\title{
Phonon dynamics in the Kitaev spin liquid
}

\author{
Mengxing Ye,,${ }^{1,2}$ Rafael M. Fernandes, ${ }^{2}$ and Natalia B. Perkins $\oplus^{2}$ \\ ${ }^{1}$ Kavli Institute for Theoretical Physics, University of California, Santa Barbara, Santa Barbara, California 93106, USA \\ ${ }^{2}$ School of Physics and Astronomy, University of Minnesota, Minneapolis, Minnesota 55455, USA
}

(Received 22 February 2020; revised 19 May 2020; accepted 6 July 2020; published 3 August 2020)

\begin{abstract}
The search for fractionalization in quantum spin liquids largely relies on their decoupling with the environment. However, the spin-lattice interaction is inevitable in a real setting. While the Majorana fermion evades a strong decay due to the gradient form of spin-lattice coupling, the study of the phonon dynamics may serve as an indirect probe of fractionalization of spin degrees of freedom. Here we propose that the signatures of fractionalization can be seen in the sound attenuation and the Hall viscosity. Despite the fact that both quantities can be related to the imaginary part of the phonon self-energy, their origins are quite different, and the time-reversal symmetry breaking is required for the Hall viscosity. First, we compute the sound attenuation due to a phonon decay by scattering with a pair of Majorana fermions and show that it is linear in temperature $(\sim T)$. We argue that it has a particular angular dependence providing the information about the spin-lattice coupling and the low-energy Majorana-fermion spectrum. The observable effects in the absence of time-reversal symmetry are then analyzed. We obtain the phonon Hall viscosity term from the microscopic Hamiltonian with time-reversal symmetry-breaking term. Importantly, the Hall viscosity term mixes the longitudinal and transverse phonon modes and renormalizes the spectrum in a unique way, which may be probed in spectroscopy measurement.
\end{abstract}

DOI: 10.1103/PhysRevResearch.2.033180

\section{INTRODUCTION}

Quantum spin liquids (QSLs), a particularly fascinating class of frustrated magnets, have been a focus of condensedmatter research since the initial proposal [1]. These systems evade magnetic order down to zero temperature and harbor a remarkable set of collective phenomena, including topological ground-state degeneracy, long-range entanglement, and fractionalized excitations [2-5]. Of particular interest is the Kitaev honeycomb model which describes a system of spin $1 / 2$ at sites of a honeycomb lattice interacting via Ising-like frustrated nearest-neighbor exchange interactions [3]. This model not only is exactly solvable with a QSL ground state, but is also realizable in real materials [6-12].

Unfortunately, the ground states of these systems cannot be easily captured by experiment, remaining featureless to conventional local probes. A promising route to detect QSLs is to look for signatures of fractionalization in dynamical probes, such as inelastic neutron scattering [13-18], Raman scattering [19-25], resonant inelastic x-ray scattering [26,27], ultrafast spectroscopy [28], and two-dimensional (2D) terahertz nonlinear coherent spectroscopy [29].

Here we propose to study the signatures of the fractionalized excitations in Kitaev materials by exploiting their coupling to lattice vibrations. Indeed, it is well known that

Published by the American Physical Society under the terms of the Creative Commons Attribution 4.0 International license. Further distribution of this work must maintain attribution to the author(s) and the published article's title, journal citation, and DOI. the coupling between collective spin and lattice degrees of freedom plays an important role in the fundamental properties of correlated materials and in many cases it has been explored rather carefully. For example, attenuation of sound due to electron-phonon coupling has been widely used to study the electronic properties and phase transitions of various solids with complex order parameters [30-32] and was proved to provide useful information on both normal and superconducting states [33-35]. The magnetoelastic coupling also plays an important role in probing vestigial phases of frustrated magnets [36]. The possibility to use the sound attenuation to study the nature of two-dimensional frustrated magnetic systems and in particular QSLs has also been recently discussed in the literature [37-40]. In particular, the spin-lattice coupling was shown to play an essential role in the theoretical interpretation of some experiments in the study of Kitaev materials, e.g., the thermal Hall transport measurement [41-43]. This suggests that, in a realistic setting, the spin-lattice coupling in Kitaev materials could be large enough to affect the phonon dynamics [40]. However, a detailed analysis of the sound attenuation in Kitaev magnets and in particular the signatures constrained by the symmetry of the spin-lattice coupled system remains mostly unexplored.

In this paper we argue that the phonon dynamics can be used to probe spin fractionalization in Kitaev materials and in particular in $\alpha-\mathrm{RuCl}_{3}[18,41,44-46]$. As a proof of principle, we study the pure Kitaev model [3] in the isotropic limit on a 2D honeycomb lattice [see Fig. 1(a)], with parameters such as sound velocity $v_{s}$ and Kitaev interaction $J_{K}$ extracted from experimental data on $\alpha-\mathrm{RuCl}_{3}[8,46]$. In the pure Kitaev model, the spins fractionalize into two types of elementary excitations: Majorana fermions and emergent gauge fluxes [3]. The 


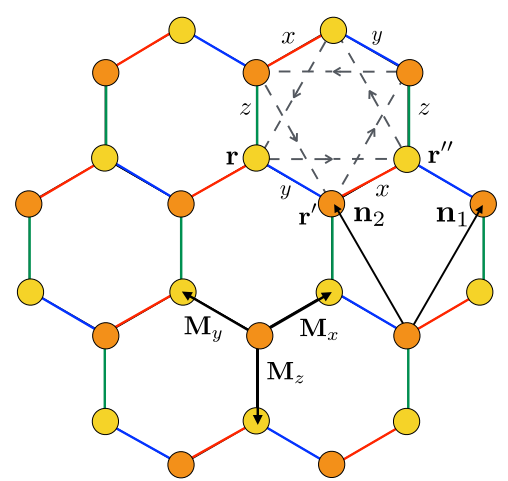

(a)

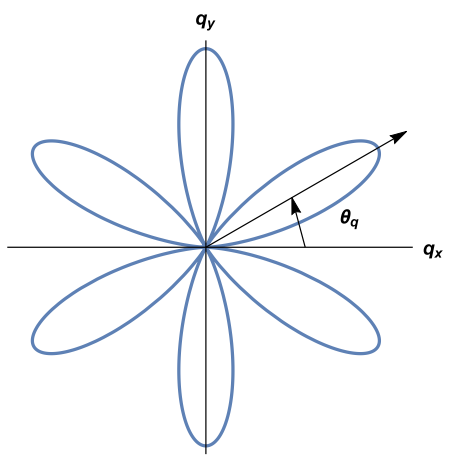

(b)

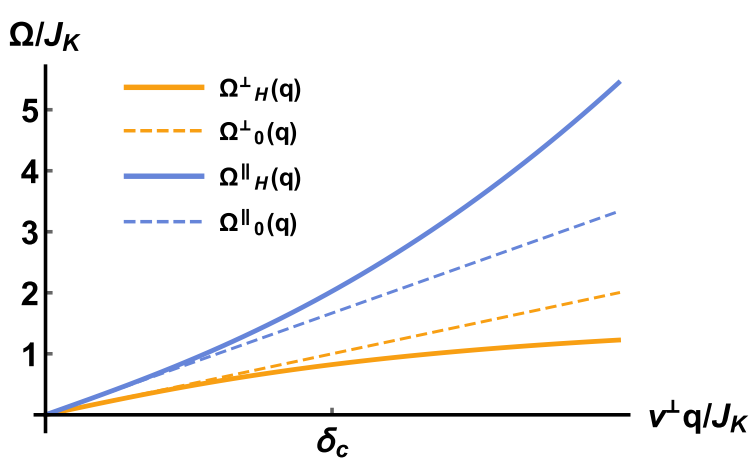

(c)

FIG. 1. (a) Kitaev model on the honeycomb lattice. The sites in the two sublattices of the honeycomb lattice $(A$ and $B)$ are marked by yellow and orange circles. Here $\mathbf{n}_{1}=\left(\frac{\sqrt{3}}{2}, \frac{3}{2}\right)$ and $\mathbf{n}_{2}=\left(-\frac{\sqrt{3}}{2}, \frac{3}{2}\right)$ are two unit vectors and we set the lattice constant $\ell_{a}=1$. The three vectors $\mathbf{M}_{x, y}=\left( \pm \frac{\sqrt{3}}{2}, \frac{1}{2}\right)$ and $\mathbf{M}_{z}=(0,-1)$ connect nearest neighbors by $x, y$, and $z$ bonds, respectively. The three sites $\mathbf{r}, \mathbf{r}^{\prime}$, and $\mathbf{r}^{\prime \prime}$ form a representative three-spin link $\left\langle\mathbf{r}, \mathbf{r}^{\prime}, \mathbf{r}^{\prime \prime}\right\rangle_{y x}$ as defined below Eq. (2). (b) Angular dependence of the sound attenuation coefficient $\alpha_{s}^{\|}$for the longitudinal mode. Here $\alpha_{s}^{\perp}$ for the transverse mode follows the same shape but is rotated by $\pi / 6$. (c) Acoustic phonon spectrum $\Omega(\mathbf{q})$. The dashed lines depict the bare spectrum with longitudinal and transverse modes. Solid lines depict the spectrum renormalized due to the mixing of transverse and longitudinal modes when time-reversal symmetry is broken. Here $\delta_{c}$ denotes the characteristic dimensionless scale beyond which the phonon spectrum is significantly bent [see Eq. (61)].

ground state of the isotropic Kitaev spin liquid corresponds to a fixed zero-flux configuration. Therefore, at temperatures below the flux gap $\Delta_{\text {flux }}$, the low-energy magnetic excitations are solely dispersive Majorana fermions. We assume that the Majorana-fermion-phonon coupling arises from the fact that the Kitaev interaction $J_{K}$ depends on the relative positions between the spins. In order to obtain an effective low-energy theory, we perform a microscopic analysis of the change of the spin exchange energy due to the lattice distortion and obtain the explicit form of the Majorana-fermion-phonon coupling by considering acoustic phonon modes coupled to low-energy spin degrees of freedom expressed in terms of Majorana fermions. We also find that in the low-energy limit, this coupling has essentially the same form as that obtained from the symmetry considerations in Ref. [39] for algebraic spin liquids.

In this picture, we study the observable consequences of the spin-lattice coupling through the phonon dynamics. By calculating the phonon self-energy, we compute the sound attenuation coefficient and the Hall viscosity, analyzing their observable signatures.

We first show that the sound attenuation is determined by the decay of a phonon due to scattering with a pair of Majorana fermions [see Fig. 2(a)], with the attenuation rate linear in temperature due to the vanishing density of states at the Dirac points. Importantly, this is the dominant process compared with the sound attenuation due to, e.g., phononphonon interactions that scale as proportional to $T^{3}$ [47]. Moreover, we find that due to the anisotropic form of the Majorana-fermion-phonon coupling and the Dirac fermionlike low-energy Hamiltonian of the magnetic excitations, the sound attenuation shows a strong angular dependence at the leading order in phonon momentum $q$ [see Fig. 1(b)]. Consequently, the angular dependence may offer a quite powerful probe of Majorana fermions with Dirac spectrum in Kitaev materials.
The same Majorana-fermion-phonon interaction also gives rise to the finite lifetime of the Majorana fermions. This effect, however, is quite weak and the lifetime scales as $\tau_{f} \sim$ Im $\Sigma_{\mathrm{MF}} \sim T^{2}$, which is much smaller than the typical fermion energy $\sim T$.

We next study the modifications of the phonon dynamics in the absence of time-reversal symmetry due to applying a magnetic field $h$. Assuming the phonons do not couple to the magnetic field directly, these corrections are induced by the spin-lattice coupling. While the sound attenuation coefficient (a)

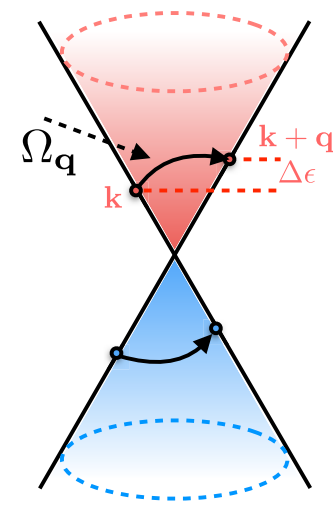

(b)

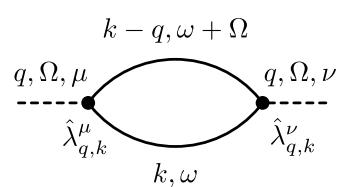

(c)

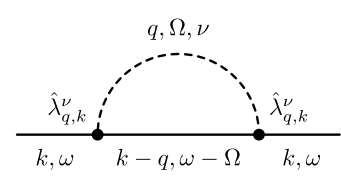

FIG. 2. (a) On-shell process in which a phonon mode with $\Omega_{\mathbf{q}}=$ $v_{s}|\mathbf{q}|$ (black dashed arrow) excites a Majorana fermion from $\mathbf{k}$ to $\mathbf{k}+\mathbf{q}$ such that $\Delta \epsilon=\epsilon_{\mathbf{k}+\mathbf{q}}-\epsilon_{\mathbf{k}}=\Omega_{\mathbf{q}}$. Red (blue) shades indicate thermal excitation (depletion) probability of Majorana fermions near a Dirac cone at $\pm K$. (b) Feynman diagram representing the phonon self-energy (36), which determines the sound attenuation and Hall viscosity for a given set of phonon polarization indices $\mu, v$. (c) Feynman diagram representing the Majorana-fermion self-energy (37) used to find the Majorana-fermion lifetime due to spin-lattice coupling. 
$\alpha_{s}$ is not changed qualitatively in a small magnetic field when $\left(h / J_{K}\right)^{3} \lesssim q \ell_{a}$, where $\ell_{a}$ is the lattice constant, the Majorana-fermion-phonon coupling introduces another interesting effect to the phonon system, i.e., a Berry phase term that mixes the transverse and longitudinal phonon modes. It is encoded in the phonon long-wavelength effective action as the Hall viscosity term, which is the leading-order term breaking the time-reversal symmetry [48,49]. It is associated with the nondissipative response to a velocity gradient of a fluid [48-50] and also contributes to the thermal Hall effect $[43,51]$. As it comes from off-shell processes, both the high-energy and low-energy Majorana-fermion modes contribute. In our analysis presented below, we calculate the Hall coefficient from the whole Majorana-fermion spectrum. We compute the nonperturbative contribution in $h$ to the Hall viscosity coefficient, which matches the result in an infinitesimally small field. We also compute the perturbative correction and show that the Hall coefficient decreases as the magnetic field strength increases. We also note that the mixing renormalizes the phonon spectrum prominently above a characteristic phonon momentum $q_{c}$ [see Fig. 1(c)] that may be observed in some spectroscopy measurements.

The rest of the paper is organized as follows. In Sec. II we present the spin-phonon Hamiltonian. First, we discuss various aspects of the extended Kitaev model (including the three-spin interaction $\kappa$ term that breaks time-reversal symmetry) that are most relevant for this study. Second, we introduce the Hamiltonian for the acoustic phonons on the honeycomb lattice. Third, we perform the symmetry analysis of the magnetoelastic coupling. The explicit microscopic derivation of the coupling vertices in terms of Majorana fermions is later presented in Sec. IV.

In Sec. III we describe the dynamics of acoustic phonons both in the presence and in the absence of the time-reversal symmetry using the long-wavelength effective action approach. To obtain the hydrodynamic coefficients from the microscopic Hamiltonian, we use the diagrammatic techniques and compute the phonon polarization bubble. Details of the calculation of the bare Green's function and self-energy are presented in Sec. V. In Sec. VI we relate the imaginary part of the diagonal components of the phonon polarization bubble to the attenuation coefficients. The phonon dynamics in the system with time-reversal-symmetry breaking is discussed in Sec. VII. We first relate the off-diagonal component of the phonon polarization bubble to the Hall viscosity coefficient and then show how it renormalizes the phonon spectrum. Both the perturbative and nonperturbative corrections in terms of $\kappa$ are obtained. A summary and a general discussion are given in Sec. VIII. Auxiliary information and technical details are provided in the Appendixes.

\section{MODEL}

We focus our discussion on the spin-phonon Hamiltonian

$$
H=H_{s}+H_{\mathrm{ph}}+H_{c} .
$$

The first term in Eq. (1) is the spin Hamiltonian given by

$$
H_{s}=-\sum_{\alpha, \mathbf{r} \in A} J^{\alpha} \sigma_{\mathbf{r}}^{\alpha} \sigma_{\mathbf{r}+\mathbf{M}_{\alpha}}^{\alpha}-\kappa \sum_{\left\langle\mathbf{r}, \mathbf{r}^{\prime}, \mathbf{r}^{\prime \prime}\right\rangle_{\alpha \gamma}} \sigma_{\mathbf{r}}^{\alpha} \sigma_{\mathbf{r}^{\prime}}^{\beta} \sigma_{\mathbf{r}^{\prime \prime}}^{\gamma},
$$

where $J^{\alpha}$ denotes the nearest-neighbor Kitaev interaction on the corresponding bond of type $\alpha=x, y, z, \sigma_{\mathbf{r}}^{\alpha}$ are the Pauli matrices, and $\mathbf{M}_{\alpha}$ labels the three inequivalent bonds on the honeycomb lattice [see Fig. 1(a)]. In the following, we ignore the presence of weak non-Kitaev couplings and weak anisotropy of the Kitaev interactions in real materials and assume $J^{\alpha}=J_{K}$ and the $C_{6 v}$ symmetry of the honeycomb lattice. The $\kappa$ term breaks time-reversal and mirror $\left(\sigma_{v}\right)$ symmetry while preserving the exact solubility of the model. The three-spin link notation $\left\langle\mathbf{r}, \mathbf{r}^{\prime}, \mathbf{r}^{\prime \prime}\right\rangle_{\alpha \gamma}$ labels bonds $\mathbf{r} \mathbf{r}^{\prime}$ and $\mathbf{r}^{\prime} \mathbf{r}^{\prime \prime}$ of types $\alpha$ and $\gamma$, respectively, on three adjacent sites $\mathbf{r}, \mathbf{r}^{\prime}$, and $\mathbf{r}^{\prime \prime}$ moving counterclockwise [see Fig. 1(a)]. Here $\alpha, \beta$, and $\gamma$ are determined such that $\beta \neq \alpha, \gamma$. It may be considered as the leading-order perturbative effect of the magnetic field in the zero-flux sector [3] and $\kappa \sim \frac{h_{x} h_{y} h_{z}}{J_{K}^{2}}$.

Decomposing spin operators into two Majorana fermions $\sigma_{\mathbf{r}}^{\alpha}=i c_{\mathbf{r}} b_{\mathbf{r}}^{\alpha}$ [3], the spin Hamiltonian can be written as

$$
\begin{aligned}
H_{s} \rightarrow \tilde{H}_{s}= & J_{K} \sum_{\alpha, \mathbf{r} \in A} i u_{\mathbf{r}, \mathbf{r}+\mathbf{M}_{\alpha}}^{\alpha} c_{\mathbf{r}} c_{\mathbf{r}+\mathbf{M}_{\alpha}} \\
& +\kappa \sum_{\left\langle\mathbf{r}, \mathbf{r}^{\prime}, \mathbf{r}^{\prime \prime}\right\rangle_{\alpha \gamma}} i u_{\mathbf{r}, \mathbf{r}^{\prime}}^{\alpha} u_{\mathbf{r}^{\prime}, \mathbf{r}^{\prime \prime}}^{\gamma} c_{\mathbf{r}} c_{\mathbf{r}^{\prime \prime}},
\end{aligned}
$$

where $u_{\mathbf{r}, \mathbf{r}+\mathbf{M}_{\alpha}}^{\alpha}=i b_{\mathbf{r}}^{\alpha} b_{\mathbf{r}+\mathbf{M}_{\alpha}}^{\alpha}, \quad \mathbf{r}^{\prime}=\mathbf{r}+\mathbf{M}_{\alpha}, \quad$ and $\quad \mathbf{r}^{\prime \prime}=\mathbf{r}+$ $\mathbf{M}_{\alpha}-\mathbf{M}_{\gamma}$. Here we use $\tilde{H}_{s}$ to label the Hamiltonian in the enlarged Hilbert space in terms of Majorana fermions. Importantly, the gauge-dependent link variables $u_{\mathbf{r}, \mathbf{r}+\mathbf{M}_{\alpha}}^{\alpha}$ are mutually commuting constants of motion in $\tilde{H}_{s}$ and they give rise to physical static $Z_{2}$-flux degrees of freedom $W_{p}=$ $\Pi_{\left\langle\mathbf{r}, \mathbf{r}+\mathbf{M}_{\alpha}\right\rangle \in p} u_{\mathbf{r}, \mathbf{r}+\mathbf{M}_{\alpha}}^{\alpha}= \pm 1$ at the plaquette $p$. Each flux sector can be characterized by a static configuration of $\left\{u_{\mathbf{r}, \mathbf{r}+\mathbf{M}_{\alpha}}^{\alpha}=\right.$ $\pm 1\}$, for which one obtains the free-fermion Hamiltonian in terms of $c_{\mathbf{r}}$. Thus, the Majorana-fermion Hamiltonian in the ground-state zero-flux sector can be obtained by setting all $u_{\mathbf{r}, \mathbf{r}+\mathbf{M}_{\alpha}}^{\alpha}=1$. In momentum space, it reads

$$
\tilde{H}_{s}=\frac{1}{2} \sum_{\mathbf{k}} \psi_{-\mathbf{k}}^{T}\left(-\tau_{x} \operatorname{Im} f_{\mathbf{k}}-\tau_{y} \operatorname{Re} f_{\mathbf{k}}+\tau_{z} \Delta_{\mathbf{k}}\right) \psi_{\mathbf{k}},
$$

where $\quad \psi_{\mathbf{k}}=\left(\frac{1}{\sqrt{2 N}} \sum_{\mathbf{r} \in A} e^{i \mathbf{k} \cdot \mathbf{r}} c_{\mathbf{r}, A}, \frac{1}{\sqrt{2 N}} \sum_{\mathbf{r} \in B} e^{i \mathbf{k} \cdot \mathbf{r}} c_{\mathbf{r}, B}\right)^{T}$ are the complex fermions obtained from the Fourier transform of the Majorana fermions $c_{A, \mathbf{r}}$ and $c_{B, \mathbf{r}}$ on sublattices $A$ and $B$, respectively ( $N$ is the number of unit cells), $\tau_{x, y}$ are the auxiliary Pauli matrices in sublattice space, $f_{\mathbf{k}}=2 J_{K}\left(1+2 \cos \sqrt{3} k_{x} / 2 e^{i 3 k_{y} / 2}\right)$ from the nearest-neighbor Majorana-fermion hopping, and $\Delta_{\mathbf{k}}=4 \kappa\left[\sin \mathbf{k} \cdot \mathbf{n}_{1}-\sin \mathbf{k}\right.$. $\left.\mathbf{n}_{2}+\sin \mathbf{k} \cdot\left(\mathbf{n}_{2}-\mathbf{n}_{1}\right)\right]$ comes from the second-neighbor Majorana hopping due to the time-reversal breaking part of the spin Hamiltonian, with $\mathbf{n}_{1}=\left(\frac{\sqrt{3}}{2}, \frac{3}{2}\right)$ and $\mathbf{n}_{2}=\left(-\frac{\sqrt{3}}{2}, \frac{3}{2}\right)$. When $\kappa=0$, the Majorana-fermion spectrum $\epsilon_{\mathbf{k}} \sim\left|f_{\mathbf{k}}\right|$ contains two Dirac points at the corners of the Brillouin zone $\pm K$.

The second term in Eq. (1) is the bare Hamiltonian for the acoustic phonons on the honeycomb lattice, which contains the kinetic and elastic energy [51,52]. The elastic part can be expressed in terms of the strain tensor $\epsilon_{i j}=\frac{1}{2}\left(\partial_{i} u_{j}+\partial_{j} u_{i}\right)$ and the elastic modulus tensor $C_{i j l k}$, where $\mathbf{u}=\left\{u_{x}, u_{y}\right\}$ is the lattice displacement vector. From symmetry considerations, for a lattice with $C_{6 v}$ point-group symmetry, there are only two independent nonzero elastic modulus tensor coefficients $C_{x x x x}$ 
and $C_{x x y y}$. The phonon Hamiltonian is written in terms of the bosonic operators that appear from quantizing the eigenmodes of the displacement vector $\mathbf{u}$. We will postpone writing down the elastic energy until next section.

The third term in Eq. (1) denotes the magnetoelastic coupling that arises from the dependence of the Kitaev coupling $J_{K}$ on the relative positions between the spins. Assuming that $J_{K}$ only depends on the distance $r$ between the atoms and that positions of the spins deviate only slightly from their equilibrium values, we expand the exchange couplings in powers of the displacement vectors. Keeping only the lowest order of this expansion, the spin-phonon interaction part of the model (1) can then be written as

$$
\begin{aligned}
H_{c} & =-\lambda \sum_{\mathbf{r}, \alpha} \mathbf{M}_{\alpha} \cdot\left[\mathbf{u}(\mathbf{r})-\mathbf{u}\left(\mathbf{r}+\mathbf{M}_{\alpha}\right)\right] \sigma_{\mathbf{r}}^{\alpha} \sigma_{\mathbf{r}+\mathbf{M}_{\alpha}}^{\alpha} \\
& =\lambda \sum_{\mathbf{r}, \alpha} \mathbf{M}_{\alpha} \cdot\left[\left(\mathbf{M}_{\alpha} \cdot \nabla\right) \mathbf{u}(\mathbf{r})\right] \sigma_{\mathbf{r}}^{\alpha} \sigma_{\mathbf{r}+\mathbf{M}_{\alpha}}^{\alpha},
\end{aligned}
$$

where $\lambda \sim\left(\frac{d J_{K}}{d r}\right)_{\mathrm{eq}} \ell_{a}$ characterizes the strength of the spinphonon interaction and $\ell_{a}$ is the lattice constant.

To find the complete set of magnetoelastic couplings, we use symmetry considerations and identify the linear combinations of the Kitaev interactions that transform as $A_{1}^{\mathrm{sp}}$ and $E_{2}^{\mathrm{sp}}$ irreducible representations (IRRs) of the $C_{6 v}$ point group. We find

$$
\begin{aligned}
A_{1}^{\mathrm{sp}} \sim & \left(\sigma_{\mathbf{r}}^{x} \sigma_{\mathbf{r}+\mathbf{M}_{x}}^{x}+\sigma_{\mathbf{r}}^{y} \sigma_{\mathbf{r}+\mathbf{M}_{y}}^{y}+\sigma_{\mathbf{r}}^{z} \sigma_{\mathbf{r}+\mathbf{M}_{z}}^{z}\right), \\
E_{2}^{\mathrm{sp}} \sim & \left\{\sigma_{\mathbf{r}}^{x} \sigma_{\mathbf{r}+\mathbf{M}_{x}}^{x}+\sigma_{\mathbf{r}}^{y} \sigma_{\mathbf{r}+\mathbf{M}_{y}}^{y}-2 \sigma_{\mathbf{r}}^{z} \sigma_{\mathbf{r}+\mathbf{M}_{z}}^{z},\right. \\
& \left.\sqrt{3}\left(\sigma_{\mathbf{r}}^{x} \sigma_{\mathbf{r}+\mathbf{M}_{x}}^{x}-\sigma_{\mathbf{r}}^{y} \sigma_{\mathbf{r}+\mathbf{M}_{y}}^{y}\right)\right\} .
\end{aligned}
$$

Similarly, in the phonon sector, the strain combinations that transform as the IRRs $A_{1}^{\mathrm{ph}}$ and $E_{2}^{\mathrm{ph}}$ are $\left(\epsilon_{x x}+\epsilon_{y y}\right)$ and $\left\{\epsilon_{x x}-\epsilon_{y y}, 2 \epsilon_{x y}\right\}$. Therefore, the spin-phonon couplings that are invariant under $C_{6 v}$ consist of two independent channels, one from $A_{1}^{\mathrm{ph}} \otimes A_{1}^{\mathrm{sp}}$ and another from $E_{2}^{\mathrm{ph}} \otimes E_{2}^{\mathrm{sp}}$, with the coupling constants $\lambda_{A_{1}}$ and $\lambda_{E_{2}}$, which might be different but of a similar strength $\lambda_{A_{1}}, \lambda_{E_{2}} \sim\left(\frac{d J_{K}}{d r}\right)_{e q} \ell_{a} \sim J_{K}$. Thus, the spin-phonon coupling Hamiltonian $H_{c}$ can be written as a sum of two independent contributions invariant under the $C_{6 v}$ symmetry, $H_{c}=H_{c}^{A_{1}}+H_{c}^{E_{2}}$, where

$$
\begin{gathered}
H_{c}^{A_{1}}=\lambda_{A_{1}} \sum_{\mathbf{r}}\left(\epsilon_{x x}+\epsilon_{y y}\right)\left(\sigma_{\mathbf{r}}^{x} \sigma_{\mathbf{r}+\mathbf{M}_{x}}^{x}+\sigma_{\mathbf{r}}^{y} \sigma_{\mathbf{r}+\mathbf{M}_{y}}^{y}+\sigma_{\mathbf{r}}^{z} \sigma_{\mathbf{r}+\mathbf{M}_{z}}^{z}\right), \\
H_{c}^{E_{2}}=\lambda_{E_{2}} \sum_{\mathbf{r}}\left[\left(\epsilon_{x x}-\epsilon_{y y}\right)\left(\sigma_{\mathbf{r}}^{x} \sigma_{\mathbf{r}+\mathbf{M}_{x}}^{x}+\sigma_{\mathbf{r}}^{y} \sigma_{\mathbf{r}+\mathbf{M}_{y}}^{y}-2 \sigma_{\mathbf{r}}^{z} \sigma_{\mathbf{r}+\mathbf{M}_{z} z}^{z}\right)\right. \\
\left.+2 \sqrt{3} \epsilon_{x y}\left(\sigma_{\mathbf{r}}^{x} \sigma_{\mathbf{r}+\mathbf{M}_{x}}^{x}-\sigma_{\mathbf{r}}^{y} \sigma_{\mathbf{r}+\mathbf{M}_{y}}^{y}\right)\right] .
\end{gathered}
$$

In Sec. IV we will use the Majorana-fermion representation of the spins to express the spin-phonon coupling $H_{c}$ in terms of the free Majorana fermion $c_{\mathbf{r}}$ and lattice displacement field $\tilde{u}_{\mathbf{q}, v}$.

\section{EFFECTIVE ACTION AND SPECTRUM FOR ACOUSTIC PHONONS}

In order to describe the dynamics of acoustic phonons both in the presence and in the absence of the time-reversal symmetry-breaking term $\kappa$, it is convenient to move away from the Hamiltonian formulation and employ instead the long-wavelength effective action $\mathcal{S}$ approach. Symmetries of the system, such as lattice symmetry and time-reversal symmetry, impose constraints on the number of nonzero independent coefficients in the elastic modulus tensor and viscosity tensor. In the following, only the nondissipative terms in the effective action are considered.

\section{A. Elastic medium with time-reversal symmetry}

We start by considering acoustic phonons in a homogeneous space medium without decay and with time-reversal symmetry. Their dynamics can be described by using the long-wavelength effective action in terms of the fields $\mathbf{u}$, which describe the displacement of an atom from its original location. To lowest order, it reads [53]

$$
\mathcal{S}_{\mathrm{ph}}^{(s)}=\int d^{2} x d \tau\left[\rho\left(\partial_{\tau} \mathbf{u}\right)^{2}+F^{(s)}\right], \quad F^{(s)}=\frac{1}{2} \mathcal{C}_{i j l k} \epsilon_{i j} \epsilon_{l k},
$$

where $\rho$ is the mass density of the lattice ions. Here the subscript in $\mathcal{S}_{\mathrm{ph}}^{(s)}$ denotes that the elastic moduli $\mathcal{C}_{i j l k}$ are symmetric under $i j \leftrightarrow l k$, and thus $F^{(s)}$ describes the symmetric part of the elastic energy. Due to the symmetry of the strain tensor $\epsilon_{i j}$ under $i \leftrightarrow j$, the elastic modulus tensor $\mathcal{C}_{i j l k}$ is also symmetric under $i \leftrightarrow j$ and $l \leftrightarrow k$. Finally, by imposing the $C_{6 v}$ lattice symmetry we are left with two independent nonzero elastic modulus tensor coefficients $C_{x x x x}$ and $C_{x x y y}$. The elastic energy can then be written as

$$
\begin{aligned}
F^{(s)}= & {\left[C_{1}\left(\epsilon_{x x}+\epsilon_{y y}\right)^{2}+C_{2}\left(\epsilon_{x x}-\epsilon_{y y}+2 i \epsilon_{x y}\right)\right.} \\
& \left.\times\left(\epsilon_{x x}-\epsilon_{y y}-2 i \epsilon_{x y}\right)\right],
\end{aligned}
$$

where $C_{1} \equiv\left(C_{x x x x}+C_{x x y y}\right) / 2$ and $C_{2} \equiv\left(C_{x x x x}-C_{x x y y}\right) / 2$. In momentum and (Matsubara) frequency space, the action is given by

$$
\mathcal{S}_{\mathrm{ph}}^{(s)}=T \sum_{\mathbf{q}, \Omega_{n}}\left(\begin{array}{cc}
u_{x,-\mathbf{q}} & u_{y,-\mathbf{q}}
\end{array}\right)\left(\begin{array}{cc}
\rho \Omega_{n}^{2}+\left(C_{1}+C_{2}\right) q_{x}^{2}+C_{2} q_{y}^{2} & C_{1} q_{x} q_{y} \\
C_{1} q_{x} q_{y} & \rho \Omega_{n}^{2}+\left(C_{1}+C_{2}\right) q_{y}^{2}+C_{2} q_{x}^{2}
\end{array}\right)\left(\begin{array}{l}
u_{x, \mathbf{q}} \\
u_{y, \mathbf{q}}
\end{array}\right) .
$$

We diagonalize Eq. (10) through

$$
\left(\begin{array}{c}
u_{x, \mathbf{q}} \\
u_{y, \mathbf{q}}
\end{array}\right)=\left(\begin{array}{cc}
\cos \theta_{q} & -\sin \theta_{q} \\
\sin \theta_{q} & \cos \theta_{q}
\end{array}\right)\left(\begin{array}{c}
\tilde{u}_{\mathbf{q}, \|} \\
\tilde{u}_{\mathbf{q}, \perp}
\end{array}\right)
$$

where $\tilde{u}_{\mathbf{q}, v}$ denotes the magnitude of lattice displacement in the $v=\|, \perp$ channels which may be quantized as the acoustic phonons. We then find the longitudinal and transverse acoustic phonon spectrum and the polarization 
vectors (defined through $\mathbf{u}_{\mathbf{q}}=\sum_{\nu} \hat{e}_{\mathbf{q}, v} \tilde{u}_{\mathbf{q}, v}$ ) to be equal to

$$
\begin{aligned}
& \Omega_{\|, \mathbf{q}}=v_{s}^{\|} q=\sqrt{\frac{C_{1}+C_{2}}{\rho}} q, \quad \hat{e}_{\mathbf{q}, \|}=\left\{\cos \theta_{q}, \sin \theta_{q}\right\}, \\
& \Omega_{\perp, \mathbf{q}}=v_{s}^{\perp} q=\sqrt{\frac{C_{2}}{\rho}} q, \quad \hat{e}_{\mathbf{q}, \perp}=\left\{-\sin \theta_{q}, \cos \theta_{q}\right\},
\end{aligned}
$$

where $q=\sqrt{q_{x}^{2}+q_{y}^{2}}$ and $\theta_{q}$ is defined in Fig. 2(b). In the case of the bare lattice system, the phonon dispersions are isotropic and the transverse and longitudinal modes are decoupled, which are generic properties for the $2 \mathrm{D}$ medium with $C_{6}$ or higher symmetries.

Another property of elastic media is the viscosity tensor, which relates the stress tensor, obtained by differentiating the action with respect to the strain tensor $\sigma_{i j}=-\frac{\delta \mathcal{S}}{\delta \epsilon_{i j}}$, to the strain rate $\sigma_{i j}=-\eta_{i j k l} \dot{\epsilon}_{k l}$. The viscosity tensor of noninteracting phonons is identically zero. However, it becomes finite when the phonon interactions that lead to phonon decay are included. In this work we consider the nonzero viscosity tensor coefficients generated only through the spin-lattice coupling. The effects due to, e.g., phonon self-interactions, are ignored for simplicity. Moreover, generally, the correction from the phonon self-interactions is subleading in temperature [49].

As the viscosity tensor coefficient coming from the phonon interactions can be anisotropic in space, i.e., dependent on $\theta_{q}$, a straightforward symmetry analysis alone does not allow us to determine the number of independent components of the symmetric viscosity tensor $\eta_{i j l k}^{(s)}$ (by the same way as we just $\operatorname{did}$ for $\left.\mathcal{C}_{i j l k}\right)$. As such, instead of finding the nonzero $\eta_{i j l k}^{(s)}$ from a symmetry analysis, here we will calculate the decay rate directly from the phonon self-energy by integrating out the Majorana fermions.

\section{B. Elastic medium with broken time-reversal symmetry}

For an elastic medium with time-reversal symmetry breaking, e.g., when $\kappa \neq 0$ in Eq. (1), there is a direct contribution to the phonon effective action coming from the Hall viscosity term $[48,49]$,

$$
\mathcal{S}_{\mathrm{ph}}^{(a)}=\int d^{2} x d t \eta_{i j l k}^{(a)} \epsilon_{i j} \dot{\epsilon}_{l k}
$$

where the viscosity tensor is antisymmetric, i.e., $\eta_{i j l k}^{(a)}=-\eta_{l k i j}^{(a)}$. In our case, the time-reversal symmetry breaking comes from the coupling of acoustic phonons with spin excitations, i.e., matter Majorana fermions with nonzero Chern number. After integrating out the fermion degrees of freedom, we are left with the phonon effective action $\mathcal{S}_{\mathrm{ph}}^{(a)}$ that breaks time-reversal symmetry.

The lattice symmetries give additional constraints on the number of independent nonzero Hall viscosity tensor elements. In our case, there is only one independent component $\eta_{x x x y}^{(a)}=\eta_{H}$. The reason is the following: The effective action must be invariant under all symmetry operations, so it must transform as the $A_{1}$ IRR. As the Hall viscosity tensor is antisymmetric, we need to look for all the antisymmetric $A_{1}$
IRRs formed by the tensor product of two phonon fields, i.e., those satisfying $\eta_{i j l k}=-\eta_{l k i j}$. For the elastic medium with $C_{6 v}$ symmetry, two phonon fields in the $E_{2}^{\mathrm{ph}}$ irreducible representation can form the tensor product given by $E_{2}^{\mathrm{ph}} \otimes E_{2}^{\mathrm{ph}} \rightarrow$ $A_{1}^{(s)}+A_{2}^{(a)}+\cdots$, where the superscript labels if the tensor is symmetric $(s)$ or antisymmetric $(a)$ under the exchange of the two phonon modes [54]. With $C_{6 v}$ symmetry, there is no antisymmetric $A_{1}$ IRR. On the other hand, the time-reversal symmetry-breaking term $\kappa$ in the spin Hamiltonian (2) lowers the symmetry from $C_{6 v}$ to $C_{6}$. As a result, the $A_{2}^{(a)}$ IRR of $C_{6 v}$ becomes the $A_{1}^{(a)}$ IRR of $C_{6}$, following the compatibility relation [55]. Consequently, we obtain one antisymmetric viscosity tensor element.

The effective action then becomes

$$
\begin{gathered}
\mathcal{S}_{\mathrm{ph}}^{(a)}=\frac{i \eta_{H}}{4} \int d x^{2} d t\left[\left(\epsilon_{x x}-\epsilon_{y y}+2 i \epsilon_{x y}\right)\left(\dot{\epsilon}_{x x}-\dot{\epsilon}_{y y}-2 i \dot{\epsilon}_{x y}\right)\right. \\
- \text { H.c. }] \\
=\int d x^{2} d t \eta_{H}\left[\left(\epsilon_{x x}-\epsilon_{y y}\right) \dot{\epsilon}_{x y}-\left(\dot{\epsilon}_{x x}-\dot{\epsilon}_{y y}\right) \epsilon_{x y}\right] .
\end{gathered}
$$

The $\mathcal{S}_{\mathrm{ph}}^{(a)}$ can also be expressed in terms of the transverse and longitudinal phonon eigenmodes $\tilde{u}_{\mathbf{q}, v}(v=\|, \perp)$ as

$$
\mathcal{S}_{\mathrm{ph}}^{(a)}=\frac{\eta_{H}}{2} \sum_{\mathbf{q}} \int d t q^{2}\left[\dot{\tilde{u}}_{\mathbf{q}, \|}(t) \tilde{u}_{-\mathbf{q}, \perp}(t)-\tilde{u}_{\mathbf{q}, \|}(t) \dot{\tilde{u}}_{-\mathbf{q}, \perp}(t)\right] .
$$

In general, the Hall viscosity coefficient $\eta_{H}$ can be found from the linear response theory through the Kubo formula, which gives [49]

$\eta_{\mu \nu}(\mathbf{q})=\lim _{\Omega \rightarrow 0} \frac{1}{\Omega} \frac{1}{\ell_{a}^{d}} \int d t e^{i \Omega t}\left\langle\left[\frac{\partial H_{c}}{\partial \tilde{u}_{\mathbf{q}, \mu}}(t), \frac{\partial H_{c}}{\partial \tilde{u}_{-\mathbf{q}, v}}(0)\right]\right)$

for an action of the form

$$
\mathcal{S}_{\mathrm{ph}}^{(a)}=\frac{1}{2} \sum_{\mathbf{q}} \int d t \eta_{\mu \nu}(\mathbf{q}) \tilde{u}_{-\mathbf{q}, \mu}(t) \dot{\tilde{u}}_{\mathbf{q}, v}(t),
$$

where we recall that $H_{c}$ is the spin-phonon coupling Hamiltonian.

As we will show in Sec. VII A, the phonon polarization bubble $\Pi_{\mathrm{ph}}^{\mu \nu}(\mathbf{q}, \Omega)$ can be expressed in a similar fashion as

$$
\Pi_{\mathrm{ph}}^{\mu v}(\mathbf{q}, \Omega)=-\frac{i}{2 !} \int d t e^{i \Omega t}\left\langle T \frac{\partial H_{c}}{\partial \tilde{u}_{\mathbf{q}, \mu}}(t) \frac{\partial H_{c}}{\partial \tilde{u}_{-\mathbf{q}, v}}(0)\right\rangle .
$$

Therefore, it is straightforward to find the relation between the response coefficient $\eta_{\mu \nu}(\mathbf{q})$ [and thus $\eta_{H}=\frac{1}{q^{2}} \eta_{\perp \|}(\mathbf{q})$ ] and $\Pi_{\mathrm{ph}}^{\mu \nu}(\mathbf{q}, \Omega)$.

\section{MICROSCOPIC DERIVATION OF THE EFFECTIVE LOW-ENERGY COUPLING HAMILTONIAN}

In this section we express the spin-lattice coupling in terms of the Majorana fermion-phonon (MFPh) coupling. To this end, we express the spin operators in Eq. (7) in terms of the Majorana fermions [3] and obtain the MFPh interaction in two 
symmetry channels

$$
\begin{aligned}
H_{c}^{A_{1}}=-i \lambda_{A_{1}} \sum_{\mathbf{r}}\left(\epsilon_{x x}+\epsilon_{y y}\right)\left(c_{\mathbf{r}, A} c_{\mathbf{r}+\mathbf{M}_{x}, B}\right. & \\
& \left.+c_{\mathbf{r}, A} c_{\mathbf{r}+\mathbf{M}_{y}, B}+c_{\mathbf{r}, A} c_{\mathbf{r}+\mathbf{M}_{z}, B}\right) \\
H_{c}^{E_{2}}=-i \lambda_{E_{2}} \sum_{\mathbf{r}} & \left(\epsilon_{x x}-\epsilon_{y y}\right)\left(c_{\mathbf{r}, A} c_{\mathbf{r}+\mathbf{M}_{x}, B}+c_{\mathbf{r}, A} c_{\mathbf{r}+\mathbf{M}_{y}, B}\right. \\
& \left.-2 c_{\mathbf{r}, A} c_{\mathbf{r}+\mathbf{M}_{z}, B}\right)+2 \sqrt{3} \epsilon_{x y}\left(c_{\mathbf{r}, A} c_{\mathbf{r}+\mathbf{M}_{x}, B}\right. \\
& \left.\left.-c_{\mathbf{r}, A} c_{\mathbf{r}+\mathbf{M}_{y}, B}\right)\right] .
\end{aligned}
$$

Since Majorana fermions satisfy $\left\{c_{\mathbf{r}, \alpha}, c_{\mathbf{r}^{\prime}, \beta}\right\}=2 \delta_{\mathbf{r}, \mathbf{r}^{\prime}} \delta_{\alpha, \beta}$ and $\left\{c_{\mathbf{k}, \alpha}, c_{\mathbf{k}^{\prime}, \beta}\right\}=\delta_{\mathbf{k},-\mathbf{k}^{\prime}} \delta_{\alpha, \beta}$, where $\alpha$ and $\beta$ denote the sublattice indices $A$ and $B$, the Fourier transformation of Majorana fermions is given by $c_{\mathbf{r}, A(B)}=\sqrt{\frac{2}{N}} \sum_{\mathbf{k}} c_{\mathbf{k}, A(B)} e^{i \mathbf{k} \cdot \mathbf{r}}$. In order to have simpler notation, in the following we define $c_{\mathbf{k}, A} \equiv a_{\mathbf{k}}$ and $c_{\mathbf{k}, B} \equiv b_{\mathbf{k}}$. Thus, the MFPh coupling Hamiltonian in momentum space becomes

$$
H_{c}=\frac{1}{\sqrt{N}} \sum_{\mathbf{q}, \mathbf{k}}\left(H_{\mathbf{q}, \mathbf{k}}^{A_{1}}+H_{\mathbf{q}, \mathbf{k}}^{E_{2}}\right),
$$

with

$$
\begin{aligned}
H_{\mathbf{q}, \mathbf{k}}^{A_{1}}= & -\frac{\lambda_{A_{1}}}{2}\left(i q_{x} u_{x, \mathbf{q}}+i q_{y} u_{y, \mathbf{q}}\right)\left(a_{-\mathbf{k}-\mathbf{q}} \quad b_{-\mathbf{k}-\mathbf{q}}\right) \\
& \times\left(-f_{\mathbf{k}}^{\prime} \tau_{y}-f_{\mathbf{k}}^{\prime \prime} \tau_{x}\right)\left(\begin{array}{c}
a_{\mathbf{k}} \\
b_{\mathbf{k}}
\end{array}\right), \\
H_{\mathbf{q}, \mathbf{k}}^{E_{2}}= & -\frac{\lambda_{E_{g}}}{2}\left(i q_{x} u_{x, \mathbf{q}}-i q_{y} u_{y, \mathbf{q}}\right)\left(\begin{array}{ll}
a_{-\mathbf{k}-\mathbf{q}} & b_{-\mathbf{k}-\mathbf{q}}
\end{array}\right) \\
& \times\left(-f_{1, \mathbf{k}}^{\prime} \tau_{y}-f_{1, \mathbf{k}}^{\prime \prime} \tau_{x}\right)\left(\begin{array}{c}
a_{\mathbf{k}} \\
b_{\mathbf{k}}
\end{array}\right)-\frac{\lambda_{E_{g}}}{2}\left(i q_{x} u_{y, \mathbf{q}}+i q_{y} u_{x, \mathbf{q}}\right) \\
& \times\left(\begin{array}{ll}
a_{-\mathbf{k}-\mathbf{q}} & \left.b_{-\mathbf{k}-\mathbf{q}}\right)\left(-f_{2, \mathbf{k}}^{\prime} \tau_{y}-f_{2, \mathbf{k}}^{\prime \prime} \tau_{x}\right)
\end{array}\left(\begin{array}{c}
a_{\mathbf{k}} \\
b_{\mathbf{k}}
\end{array}\right) .\right.
\end{aligned}
$$

Here we used the fact that $\epsilon_{i j} \rightarrow \frac{i}{2}\left(q_{i} u_{j}+q_{j} u_{i}\right)$ and kept only leading in $\mathbf{q}$ terms. The auxiliary Pauli matrices $\tau_{x}$ and $\tau_{y}$ in sublattice space are again used in order to write the MFPh coupling in the matrix form. Note that we used a prime (double prime) to denote the real (imaginary) part. We also defined

$$
\begin{gathered}
f_{\mathbf{k}}=2 J\left(e^{i \mathbf{k} \cdot \mathbf{n}_{1}}+e^{i \mathbf{k} \cdot \mathbf{n}_{2}}+1\right), \\
f_{1, \mathbf{k}}=2 J\left(e^{i \mathbf{k} \cdot \mathbf{n}_{1}}+e^{i \mathbf{k} \cdot \mathbf{n}_{2}}-2\right), \\
f_{2, \mathbf{k}}=2 \sqrt{3} J\left(e^{i \mathbf{k} \cdot \mathbf{n}_{1}}-e^{i \mathbf{k} \cdot \mathbf{n}_{2}}\right) .
\end{gathered}
$$

In order to obtain the MFPh coupling vertices, we express the phonon modes in terms of the transverse and longitudinal eigenmodes defined in Eq. (11). This gives

$$
\begin{aligned}
& H_{\mathbf{q}, \mathbf{k}}^{\|}=\tilde{u}_{\mathbf{q}, \|}\left(a_{-\mathbf{k}-\mathbf{q}} \quad b_{-\mathbf{k}-\mathbf{q}}\right) \hat{\lambda}_{\mathbf{q}, \mathbf{k}}^{\|}\left(\begin{array}{l}
a_{\mathbf{k}} \\
b_{\mathbf{k}}
\end{array}\right), \\
& H_{\mathbf{q}, \mathbf{k}}^{\perp}=\tilde{u}_{\mathbf{q}, \perp}\left(\begin{array}{ll}
a_{-\mathbf{k}-\mathbf{q}} & b_{-\mathbf{k}-\mathbf{q}}
\end{array}\right) \hat{\lambda}_{\mathbf{q}, \mathbf{k}}^{\perp}\left(\begin{array}{c}
a_{\mathbf{k}} \\
b_{\mathbf{k}}
\end{array}\right),
\end{aligned}
$$

where the MFPh vertices are

$$
\begin{gathered}
\hat{\lambda}_{\mathbf{q}, \mathbf{k}}^{\|}=\frac{i \lambda_{A_{1}}}{2} q\left(f_{\mathbf{k}}^{\prime} \tau_{y}+f_{\mathbf{k}}^{\prime \prime} \tau_{x}\right)+\frac{i \lambda_{E_{2}}}{2} q\left[\cos 2 \theta_{q}\left(f_{1, \mathbf{k}}^{\prime} \tau_{y}+f_{1, \mathbf{k}}^{\prime \prime} \tau_{x}\right)\right. \\
\left.+\sin 2 \theta_{q}\left(f_{2, \mathbf{k}}^{\prime} \tau_{y}+f_{2, \mathbf{k}}^{\prime \prime} \tau_{x}\right)\right], \\
\begin{aligned}
\hat{\lambda}_{\mathbf{q}, \mathbf{k}}^{\perp}=\frac{i \lambda_{E_{2}}}{2} q[ & -\sin 2 \theta_{q}\left(f_{1, \mathbf{k}}^{\prime} \tau_{y}+f_{1, \mathbf{k}}^{\prime \prime} \tau_{x}\right) \\
+ & \left.\cos 2 \theta_{q}\left(f_{2, \mathbf{k}}^{\prime} \tau_{y}+f_{2, \mathbf{k}}^{\prime \prime} \tau_{x}\right)\right] .
\end{aligned}
\end{gathered}
$$

To the leading order in momentum $q$ and near the Dirac points $\pm K$ of the Majorana-fermion spectrum, the MFPh vertices $\hat{\lambda}_{\mathbf{q}, \mathbf{k}}^{v}$ are given by

$$
\begin{aligned}
& \hat{\lambda}_{\mathbf{q}, \pm K+\mathbf{k}}^{\|} \equiv \hat{\lambda}_{\mathbf{q}, \mathbf{k}}^{\|}=3 i q \lambda_{E_{2}}\left( \pm \sin 2 \theta_{q} \tau^{x}-\cos 2 \theta_{q} \tau^{y}\right), \\
& \hat{\lambda}_{\mathbf{q}, \pm K+\mathbf{k}}^{\perp} \equiv \hat{\lambda}_{\mathbf{q}, \mathbf{k}}^{\perp}=3 i q \lambda_{E_{2}}\left( \pm \cos 2 \theta_{q} \tau^{x}+\sin 2 \theta_{q} \tau^{y}\right),
\end{aligned}
$$

where now $\mathbf{k}$ denotes the deviation from the Dirac point. Since the MFPh coupling in the $A_{1}$ channel appears at higher orders in $\mathbf{k}$, whenever the computation is restricted to the low-energy Majorana fermions near the $\pm K$ points, the $A_{1}$ channel is ignored and only the dominant contribution in the $E_{2}$ channel is considered.

\section{PHONON PROPAGATOR}

Our next task is to integrate out the Majorana fermions and obtain an effective low-energy theory only for the phonons.

\section{A. Free-phonon propagator}

The free-phonon propagator in terms of lattice displacement field $\tilde{u}_{\mathbf{q}, v}$ defined in Eq. (11) is given by

$$
D_{v \nu, \mathbf{q}}^{(0)}(t)=-i\left\langle T \tilde{u}_{-\mathbf{q}, v}(t) \tilde{u}_{\mathbf{q}, v}(0)\right\rangle^{(0)},
$$

where the superscript $(0)$ denotes the bare propagator and $v=\|, \perp$ labels the polarization. The second quantized form of $\tilde{u}_{\mathbf{q}, v}(t)$ is given by [56]

$$
\tilde{u}_{\mathbf{q}, v}(t)=i\left(\frac{\hbar}{2 \rho \delta_{V} \Omega_{\mathbf{q}}}\right)^{1 / 2}\left(a_{\mathbf{q}} e^{-i \Omega_{\mathbf{q}} t}+a_{-\mathbf{q}}^{\dagger} e^{i \Omega_{\mathbf{q}} t}\right),
$$

where $\delta_{V}$ is the area enclosed in one unit cell and $\rho$ is the mass density of the lattice ions. The time-ordered phonon propagator in the momentum and frequency space is then given by

$$
D_{\nu \nu}^{(0)}(\mathbf{q}, \Omega)=\int d t D_{v v, \mathbf{q}}^{(0)}(t) e^{i \Omega t}=-\frac{\hbar}{\rho \delta_{V}} \frac{1}{\Omega^{2}-\Omega_{\mathbf{q}}^{2}+i \delta} .
$$

In the rest of the discussion we set $\hbar=1$.

\section{B. Phonon polarization bubble}

To compute the corrections to the effective phonon action due to the spin-lattice coupling, we calculate the phonon oneloop self-energy shown in Fig. 2(b) that can be expressed as

$$
\begin{aligned}
\Pi_{\mathrm{ph}}^{\mu \nu}(\mathbf{q}, \Omega)= & \frac{-i}{2 !} \int d t e^{i \Omega t} \frac{1}{N} \sum_{\mathbf{k}, \mathbf{k}^{\prime}}\left\langle T \psi_{-\mathbf{k}-\mathbf{q}}^{T}(t) \hat{\lambda}_{\mathbf{q}, \mathbf{k}}^{\mu} \psi_{\mathbf{k}}(t)\right. \\
& \left.\times \psi_{-\mathbf{k}^{\prime}+\mathbf{q}}^{T}(0) \hat{\lambda}_{-\mathbf{q}, \mathbf{k}^{\prime}}^{v} \psi_{\mathbf{k}^{\prime}}(0)\right\rangle \\
= & i \operatorname{Tr}\left[\hat{\lambda}_{\mathbf{q}, \mathbf{k}}^{v} \mathcal{G}(\mathbf{k}, \omega) \hat{\lambda}_{\mathbf{q}, \mathbf{k}}^{\mu} \mathcal{G}(\mathbf{k}-\mathbf{q}, \omega+\Omega)\right],
\end{aligned}
$$


where $\mu, v=\|, \perp$ and $\mathcal{G}(\mathbf{k}, \omega)$ denotes the Majorana-fermion Green's function given by

$$
\begin{aligned}
\mathcal{G}(\mathbf{k}, \omega)= & -i \int_{-\infty}^{+\infty} d t\left\langle T \psi_{\mathbf{k}}(t) \psi_{-\mathbf{k}}^{T}(0)\right| e^{i \omega t} \\
= & \frac{1}{2}\left[\frac{1}{\omega+\left|\overrightarrow{\mathcal{F}}_{\mathbf{k}}\right|-i \delta}\left(I-\frac{\overrightarrow{\mathcal{F}}_{\mathbf{k}} \cdot \vec{\tau}}{\left|\overrightarrow{\mathcal{F}}_{\mathbf{k}}\right|}\right)\right. \\
& \left.+\frac{1}{\omega-\left|\overrightarrow{\mathcal{F}}_{\mathbf{k}}\right|+i \delta}\left(I+\frac{\overrightarrow{\mathcal{F}}_{\mathbf{k}} \cdot \vec{\tau}}{\left|\overrightarrow{\mathcal{F}}_{\mathbf{k}}\right|}\right)\right] \\
= & \frac{1}{2} \sum_{s= \pm} \frac{1}{\omega+s\left|\overrightarrow{\mathcal{F}}_{\mathbf{k}}\right|-s i \delta}\left[I-s \frac{\overrightarrow{\mathcal{F}}_{\mathbf{k}} \cdot \vec{\tau}}{\left|\overrightarrow{\mathcal{F}}_{\mathbf{k}}\right|}\right],
\end{aligned}
$$

where $\overrightarrow{\mathcal{F}}_{\mathbf{k}}=\left\{-\operatorname{Im} f_{\mathbf{k}},-\operatorname{Re} f_{\mathbf{k}}, \Delta_{\mathbf{k}}\right\}$ [see Appendix A for details of the derivation]. Recall that $\Delta_{\mathbf{k}}=4 \kappa\left[\sin \mathbf{k} \cdot \mathbf{n}_{1}-\right.$ $\sin \mathbf{k} \cdot \mathbf{n}_{2}+\sin \mathbf{k} \cdot\left(\mathbf{n}_{1}-\mathbf{n}_{2}\right)$ ] is only nonzero when timereversal symmetry is broken. We also use $\hat{\lambda}_{\mathbf{q}, \mathbf{k}-\mathbf{q}}=\hat{\lambda}_{\mathbf{q}, \mathbf{k}}+$ $O\left(q^{2}\right)$ and drop the subleading $O\left(q^{2}\right)$ terms. In Eq. (32), $\operatorname{Tr}[\cdots]$ sums over momentum $\mathbf{k}$ and sublattice degrees of freedom $\left(A\right.$ and $B$ ) and integrates over frequency as $\int \frac{d \omega}{2 \pi}$. Note that since the Majorana fermions are real, the Majorana fermionic excitations at $(\mathbf{k}, \omega)$ and $(-\mathbf{k},-\omega)$ are physically the same, so $\mathcal{G}(\mathbf{k}, \omega)=-\mathcal{G}(-\mathbf{k},-\omega)^{T}$. We also note that since our computation is performed directly in terms of Majorana fermions, all $\mathbf{k}$ and $\omega$ modes should be summed over, which is different from the calculations that are performed in terms of the complex-fermion representation.

The renormalized phonon propagator is then given by the equation

$$
D(\mathbf{q}, \Omega)=\left\{\left[D^{(0)}(\mathbf{q}, \Omega)\right]^{-1}-\Pi_{\mathrm{ph}}(\mathbf{q}, \Omega)\right\}^{-1} .
$$

For calculations at finite temperature $T$, it is convenient to express the Majorana-fermion Green's function and the phonon polarization bubble in the Matsubara frequency representation

$$
\mathcal{G}\left(\mathbf{k}, i \omega_{m}\right)=\frac{1}{2} \sum_{s= \pm} \frac{1}{i \omega_{m}+s\left|\overrightarrow{\mathcal{F}}_{\mathbf{k}}\right|}\left(I-s \frac{\overrightarrow{\mathcal{F}}_{\mathbf{k}} \cdot \vec{\tau}}{\left|\overrightarrow{\mathcal{F}}_{\mathbf{k}}\right|}\right)
$$

and

$$
\begin{aligned}
& \Pi_{\mathrm{ph}}^{\mu \nu}\left(\mathbf{q}, i \Omega_{n}\right) \\
& \quad=-\operatorname{Tr}\left[\hat{\lambda}_{\mathbf{q}, \mathbf{k}}^{v} \mathcal{G}\left(\mathbf{k}, i \omega_{m}\right) \hat{\lambda}_{\mathbf{q}, \mathbf{k}}^{\mu} \mathcal{G}\left(\mathbf{k}-\mathbf{q}, i\left(\omega_{m}+\Omega_{n}\right)\right)\right],
\end{aligned}
$$

where $\operatorname{Tr}[\cdots]$ now sums over momentum $\mathbf{k}$, Matsubara frequencies $i \omega_{m}$ as $T \sum_{m}$, and sublattice degrees of freedom. In general, the phonon polarization bubble $\Pi_{\mathrm{ph}}$ contributes to the renormalization of the sound velocity [52], mixing of the transverse and longitudinal phonon modes [49], and attenuation and absorption of sound waves [38,39]. We compute these effects explicitly in Secs. VI and VII.

As a final remark, we note that the corrections to the spin excitation dynamics from the spin-lattice coupling are relatively weak. The Majorana-fermion self-energy [Fig. 2(c)] can be expressed as

$$
\Sigma(\mathbf{k}, \omega)=i \operatorname{Tr}\left[\hat{\lambda}_{\mathbf{q}, \mathbf{k}}^{v} D_{v v}^{(0)}(\mathbf{q}, \Omega) \hat{\lambda}_{\mathbf{q}, \mathbf{k}}^{v} \mathcal{G}(\mathbf{k}-\mathbf{q}, \omega-\Omega)\right],
$$

where the bare phonon propagator in terms of the lattice displacement field $\tilde{u}_{\mathbf{q}, v}$ is given by Eq. (29). Here again we only keep terms linear in $\mathbf{q}$ and $\mathbf{k}$ in the MFPh coupling vertices, so $\hat{\lambda}_{\mathbf{q}, \mathbf{k}-\mathbf{q}} \simeq \hat{\lambda}_{\mathbf{q}, \mathbf{k}}$. In addition, $\operatorname{Tr}[\cdots]$ sums over momentum $\mathbf{q}$ and polarization $v$ and integrates over the phonon frequency as $\int \frac{d \Omega}{2 \pi}$. From Eq. (37) we find the imaginary part of $\Sigma \sim T^{2}$, which is smaller than the typical fermion energy $\sim T$.

\section{ANALYTICAL CALCULATION OF ATTENUATION COEFFICIENT}

The quantitative description of the attenuation process can be obtained through the lossy acoustic wave function which decays with distance away from the driving source as

$$
\mathbf{u}(\mathbf{x}, t)=\mathbf{u}_{0} e^{-\alpha_{s}(\mathbf{q}) x} e^{i(\Omega t-\mathbf{q} \cdot \mathbf{x})},
$$

where $\mathbf{u}(\mathbf{x}, t)$ is the lattice displacement vector, $\mathbf{u}_{0}=\mathbf{u}(\mathbf{x}, t=$ $0), \Omega$ is the acoustic wave frequency, and $\mathbf{q}$ is the propagation vector. The attenuation coefficient $\alpha_{s}(\mathbf{q})$, defined as the inverse of the phonon mean free path, can be calculated from the imaginary part of the phonon self-energy as

$$
\alpha_{s}(\mathbf{q}) \propto-\frac{1}{v_{s}^{2} q} \operatorname{Im}\left[\Pi_{\mathrm{ph}}(\mathbf{q}, \Omega)\right]_{\Omega=v_{s} q},
$$

where $v_{s}$ is the sound velocity. The derivation of this result is shown in Appendix B.

\section{A. Sound attenuation coefficient at $\boldsymbol{T}>\boldsymbol{0}$}

The lowest-order Majorana-fermion-phonon interaction leading to the phonon damping is shown diagrammatically in Fig. 2(b) with $\mu=v$. In this section we will evaluate the sound attenuation coefficient $\alpha_{s}(q)$ by relating it to the imaginary part of the diagonal components of the polarization bubble $\operatorname{Im} \Pi_{\mathrm{ph}}^{v v}(\mathbf{q}, \Omega)$ defined in Eq. (32). We will consider the sound attenuation in the temperature range $v_{F} q<T<\Delta_{\text {flux }}$, where $v_{F}$ is the Majorana-fermion velocity at the Dirac point, and assume $v_{s}<v_{F}$, the situation which can be potentially realized in Kitaev materials $[8,46]$. Under these assumptions, there is a finite phase space for the scattering of phonons with thermally excited low-energy Majorana fermions [see Fig. 2(a)].

We first note that from the kinematic constraints, $\operatorname{Im} \Pi_{\mathrm{ph}}^{v v}(q, \Omega) \neq 0$ only at $T>0$ when $v_{s}<v_{F}$. To compute $\Pi_{\mathrm{ph}}^{v v}(\mathbf{q}, \Omega)$ at finite temperature, it is convenient to first evaluate in the Matsubara frequency representation, i.e., $\Pi_{\mathrm{ph}}^{v v}\left(\mathbf{q}, i \Omega_{n}\right)$ in Eq. (36), and then perform the analytical continuation to the real frequency. Note that the dynamical part only appears in the denominator of the Majorana-fermion Green's function [see Eq. (35)]. Thus, we can first evaluate the frequency summation [57]

$$
\begin{aligned}
\mathbb{P}^{s, s^{\prime}}\left(\mathbf{k}, \mathbf{q}, i \Omega_{n}\right) & \equiv T \sum_{m} \frac{1}{i \omega_{m}+s\left|\overrightarrow{\mathcal{F}}_{\mathbf{k}}\right|} \frac{1}{i\left(\omega_{m}+\Omega_{n}\right)+s^{\prime}\left|\overrightarrow{\mathcal{F}}_{\mathbf{k}-\mathbf{q}}\right|} \\
& =\frac{1}{2} \frac{s \tanh \beta\left|\overrightarrow{\mathcal{F}}_{\mathbf{k}}\right| / 2-s^{\prime} \tanh \beta\left|\overrightarrow{\mathcal{F}}_{\mathbf{k}-\mathbf{q}}\right| / 2}{i \Omega_{n}+s^{\prime}\left|\overrightarrow{\mathcal{F}}_{\mathbf{k}-\mathbf{q}}\right|-s\left|\overrightarrow{\mathcal{F}}_{\mathbf{k}}\right|},
\end{aligned}
$$

where $s$ and $s^{\prime}$ correspond to the \pm signs in the Majorana-fermion Green's function $\mathcal{G}\left(\mathbf{k}, i \omega_{m}\right)$ [see Eq. (35)]. Performing the analytical continuation to the real frequency, 
we get

$\mathbb{P}^{s, s^{\prime}}(\mathbf{k}, \mathbf{q}, \Omega)=\frac{1}{2} \frac{s \tanh \beta\left|\overrightarrow{\mathcal{F}}_{\mathbf{k}}\right| / 2-s^{\prime} \tanh \beta\left|\overrightarrow{\mathcal{F}}_{\mathbf{k}-\mathbf{q}}\right| / 2}{\Omega+s^{\prime}\left|\overrightarrow{\mathcal{F}}_{\mathbf{k}-\mathbf{q}}\right|-s\left|\overrightarrow{\mathcal{F}}_{\mathbf{k}}\right|+i \delta \operatorname{sgn} \Omega}$.

Note that $\operatorname{Im} \mathbb{P}^{s, s^{\prime}}(\mathbf{k}, \mathbf{q}, \Omega) \neq 0$ only when there is a pole in the denominator of Eq. (41), i.e., $\Omega+s^{\prime}\left|\overrightarrow{\mathcal{F}}_{\mathbf{k}-\mathbf{q}}\right|-s\left|\overrightarrow{\mathcal{F}}_{\mathbf{k}}\right|=0$, which for $v_{s}<v_{F}$ requires the same choice of the sign in $s$ and $s^{\prime}$, i.e., $s s^{\prime}>0$. Moreover, when $s s^{\prime}>0$, the numerator of Eq. (41) is finite only when $\left|\overrightarrow{\mathcal{F}}_{\mathbf{k}}\right|,\left|\overrightarrow{\mathcal{F}}_{\mathbf{k}-\mathbf{q}}\right| \lesssim T$ and is exponentially suppressed when $\left|\overrightarrow{\mathcal{F}}_{\mathbf{k}}\right|,\left|\overrightarrow{\mathcal{F}}_{\mathbf{k}-\mathbf{q}}\right| \gtrsim 1 / \beta \sim T$.

In the temperature range of our interest $T<\Delta_{\text {flux }} \ll J_{K}$, the spectrum of the Majorana fermions can be linearized near the Dirac points $\pm K$ and the MFPh vertices $\hat{\lambda}_{\mathbf{q}, \mathbf{k}}^{v}$ are given by Eq. (28). In this approximation, the MFPh vertices are constant in terms of $\mathbf{k}$ and thus the momentum summation in Eq. (36) can be obtained by first replacing the true MFPh vertices with the Pauli matrices $\tau^{x, y}$. We then get

$$
\begin{aligned}
\tilde{\Pi}_{\alpha \beta}(\mathbf{q}, \Omega) \equiv & \tilde{\Pi}_{\alpha \beta}=\operatorname{Tr}\left[\tau^{\alpha} \mathcal{G}\left(\mathbf{k}, i \omega_{m}\right)\right. \\
& \left.\times \tau^{\beta} \mathcal{G}\left(\mathbf{k}-\mathbf{q}, i\left(\omega_{m}+\Omega_{n}\right)\right)\right]_{i \Omega_{n} \rightarrow \Omega+i \delta},
\end{aligned}
$$

where $\alpha, \beta=x, y$. The explicit expressions for $\tilde{\Pi}_{x x}, \tilde{\Pi}_{y y}$, and $\tilde{\Pi}_{x y}$ are derived in Appendix C.

The diagonal components of $\operatorname{Im} \Pi_{\mathrm{ph}}^{v v}(\mathbf{q}, \Omega)$ can now be expressed in a straightforward way in terms of $\tilde{\Pi}_{\alpha \beta}$ as

$$
\begin{aligned}
& \Pi_{\mathrm{ph}}^{\|\|}(\mathbf{q}, \Omega)=9 \lambda_{E_{2}}^{2} q^{2}[ \sin ^{2} 2 \theta_{q} \tilde{\Pi}_{x x}+\cos ^{2} 2 \theta_{q} \tilde{\Pi}_{y y} \\
&\left.-\sin 2 \theta_{q} \cos 2 \theta_{q}\left(\tilde{\Pi}_{x y}+\tilde{\Pi}_{y x}\right)\right], \\
& \Pi_{\mathrm{ph}}^{\perp \perp}(\mathbf{q}, \Omega)=9 \lambda_{E_{2}}^{2} q^{2} {\left[\cos ^{2} 2 \theta_{q} \tilde{\Pi}_{x x}+\sin ^{2} 2 \theta_{q} \tilde{\Pi}_{y y}\right.} \\
&\left.+\sin 2 \theta_{q} \cos 2 \theta_{q}\left(\tilde{\Pi}_{x y}+\tilde{\Pi}_{y x}\right)\right] .
\end{aligned}
$$

In the presence of time-reversal symmetry, i.e., when $\kappa=0$, which corresponds to the $z$ component of $\overrightarrow{\mathcal{F}}_{\mathbf{k}}$ vanishing, we find that the imaginary parts of the phonon polarization bubble for the longitudinal and transverse polarizations are (see Appendix $\mathrm{C}$ for derivation)

$$
\begin{gathered}
\operatorname{Im} \Pi_{\mathrm{ph}}^{\|\| \|}(\mathbf{q}, \Omega) \approx-\frac{36 \pi \lambda_{E_{2}}^{2} q|\Omega|}{v_{F}^{3} \mathcal{A}_{\mathrm{BZ}}} T\left(1-\cos 6 \theta_{q}\right) \ln 2, \\
\operatorname{Im} \Pi_{\mathrm{ph}}^{\perp \perp}(\mathbf{q}, \Omega) \approx-\frac{36 \pi \lambda_{E_{2}}^{2} q|\Omega|}{v_{F}^{3} \mathcal{A}_{\mathrm{BZ}}} T\left(1+\cos 6 \theta_{q}\right) \ln 2 .
\end{gathered}
$$

In a system with time-reversal symmetry breaking, from Eqs. (3) and (4) we note that the $\kappa$ term does not excite the $Z_{2}$ flux but opens a gap in the Majorana-fermion bands with Chern number \pm 1 [3]. The correction to the sound attenuation is perturbative in $\kappa$. Moreover, when the gap at $\pm K$ is small, i.e., $\kappa<v_{F} q$, the correction is negligible. We do not consider this correction to the sound attenuation further.

To understand how the constraint $v_{F} q<T<\Delta_{\text {flux }}$ is imposed in the computation, we first note that when $v_{s}<v_{F}$, the thermally excited Majorana fermions that can scatter off of the acoustic phonon should have minimum energy $\sim v_{F} q / 2$. To optimize the phase space for phonon decay, we require $v_{F} q \ll T$. On the other hand, when $T \sim \Delta_{\text {flux }}$, the thermally excited flux degrees of freedom should be important and the zero-flux sector approximation breaks down. These processes remain to be considered in future work.
From Eq. (B8) we find the sound attenuation coefficient to be equal to

$$
\alpha_{s}^{v}(\mathbf{q}) \sim\left(\frac{\lambda_{E_{2}}}{v_{F} / \ell_{a}}\right)^{2} \frac{v_{s}^{v}}{v_{F}} \frac{T}{C_{\nu} \delta_{V}} q\left(1 \mp \cos 6 \theta_{q}\right),
$$

where $C_{\nu} \delta_{V}=m_{\text {ion }}\left(v_{s}^{v}\right)^{2}$ is determined by the elastic modulus tensor coefficient of the material, where $C_{\|}=C_{1}+C_{2}, C_{\perp}=C_{2}$, and the upper and lower signs are for $v=\|$ and $v=\perp$ polarizations, respectively. Importantly, $1 \mp \cos 6 \theta_{q}$ is highly anisotropic and vanishes along certain directions, as shown in Fig. 1(b). We note that the $\alpha_{s}$ due to the phonon-Majoranafermion coupling should be much larger than that from the phonon anharmonic interaction. As $\frac{\lambda_{E_{2}}}{v_{F} / \ell_{a}} \sim 1$ and $\frac{v_{s}^{v}}{v_{F}} \sim 1$, the main suppression of $\alpha_{s}$ comes from the ratio $T / C_{\nu} \delta V$, where $C_{\nu} \delta V$ should be a large energy of order of eV. On the other hand, as $C_{\nu} \delta V$ appears for a generic sound attenuation mechanism, at low $T, \alpha_{s, \mathrm{ph}-\mathrm{ph}} / \alpha_{s, \mathrm{ph}-\mathrm{MF}} \sim\left(\frac{T}{v_{s} / \ell_{a}}\right)^{2} \ll 1$, so the sound attenuation due to Majorana-fermion-phonon coupling is dominant.

We note two important features of the attenuation coefficient. First, $\alpha_{s}$ scales linearly with temperature, which counts the phase space of the Majorana-fermion scattering. Second, while the phonon spectrum is isotropic at the leading $q^{2}$ order due to the sixfold rotation symmetry of the lattice, $\operatorname{Im}\left[\Pi_{\mathrm{ph}}^{v v}(\mathbf{q}, \Omega)\right]$ can be anisotropic due to the coupling to the fermions with the Dirac spectrum. The angular modulation factor $\cos 6 \theta_{q}$ may be understood from symmetry considerations as follows. The phonon polarization bubble [see Eq. (32)] is a convolution of two MFPh coupling vertices (each in the $E_{2}^{\mathrm{ph}} \otimes E_{2}^{\mathrm{sp}}$ representation) and two Majoranafermion propagators [each $\mathcal{G}$ near the Dirac points in Eq. (33) contains an $\left.E_{1}^{\mathrm{MF}} \mathrm{IRR}\right]$. So $\alpha_{s}$, in the identity $\operatorname{IRR}\left(A_{1}\right)$, comes as the product of $E_{2}^{\mathrm{ph}} \otimes E_{2}^{\mathrm{sp}} \otimes E_{1}^{\mathrm{MF}} \otimes E_{2}^{\mathrm{ph}} \otimes E_{2}^{\mathrm{sp}} \otimes E_{1}^{\mathrm{MF}}$ in Eq. (32), which gives an angular modulation $\cos 6 \theta_{q}$.

\section{PHONON DYNAMICS WITH TIME-REVERSAL SYMMETRY BREAKING}

In this section we will study the observable consequences of the Majorana-fermion-phonon interaction in the presence of time-reversal symmetry breaking, for example, due to an applied magnetic field. As was discussed above, the leadingorder perturbation from the magnetic field is the three-spin interaction $\kappa$ term in the spin Hamiltonian, which does not change the sound attenuation coefficient qualitatively. On the other hand, the phonon system experiences Berry curvature induced by the $\kappa$ term due to the spin-lattice coupling. In terms of the effective action, this is the Hall viscosity term given by Eq. (13). Here we first compute the Hall viscosity coefficient by relating it to the off-diagonal component of the polarization bubble that is odd under time reversal and then show how it renormalizes the phonon spectrum.

\section{A. Hall viscosity coefficient with time-reversal symmetry breaking}

We start by deriving the Hall viscosity coefficient $\eta_{H}$, which we introduced in Sec. IIIB. We first recall that it comes from the imaginary part of the off-diagonal component 
of the phonon polarization bubble, which is antisymmetric in exchanging the polarization indices $\mu \nu$. This process is nondissipative as only the symmetric part of the viscosity coefficients contributes to dissipation [48-50,53]. Consequently, $\operatorname{Im} \Pi_{\mathrm{ph}}(\mathbf{q}, \Omega)$ does not necessarily come from the pole in $\Pi_{\mathrm{ph}}(\mathbf{q}, \Omega)$, which involves on-shell scattering and is generally dissipative.

In the following, we consider the contribution when onshell processes are not involved, which is called the intrinsic contribution in the study of the anomalous Hall effect [58]. It turns out that the intrinsic contribution and the scattering contribution can be separated when $v_{s}<v_{F}$. Whereas the intrinsic contribution is nonzero at $T=0$, the scattering contribution requires $T>0$ for the reason we discussed in Sec. VI A. We also note that as there is no kinematic constraint for the intrinsic contribution, both the low-energy and high-energy Majorana fermions contribute to the polarization bubble.

In the following, we restrict our analysis to $T=0$. From Eqs. (32) and (33) we note that at $T=0$ only the convolution of fermion propagators with $s s^{\prime}<0$ is nonzero. Similarly to how the sound attenuation coefficient was obtained in the preceding section, it is convenient to integrate over frequencies first. We obtain

$$
\begin{aligned}
\mathbb{P}^{ \pm, \mp}(\mathbf{k}, \mathbf{q}, i \Omega) & =\int \frac{d \omega}{2 \pi} \frac{1}{i \omega \pm\left|\overrightarrow{\mathcal{F}}_{\mathbf{k}}\right|} \frac{1}{i(\omega+\Omega) \mp\left|\overrightarrow{\mathcal{F}}_{\mathbf{k}-\mathbf{q}}\right|} \\
& =\frac{1}{i \Omega \mp\left(\left|\overrightarrow{\mathcal{F}}_{\mathbf{k}-\mathbf{q}}\right|+\left|\overrightarrow{\mathcal{F}}_{\mathbf{k}}\right|\right)} .
\end{aligned}
$$

Next we compute the off-diagonal component of the polarization bubble. The MFPh coupling follows from Eqs. (26) and (27). To compute the polarization bubble, it is again convenient to first compute

$$
\tilde{\Pi}^{\alpha \beta}(\mathbf{q}, \mathbf{k}, i \Omega)=\operatorname{Tr}\left[\tau^{\alpha} \mathcal{G}(\mathbf{k}, i \omega) \tau^{\beta} \mathcal{G}(\mathbf{k}-\mathbf{q}, i(\omega+\Omega))\right],
$$

where $\tau^{\alpha, \beta}$ are Pauli matrices in sublattice space and $\operatorname{Tr}[\cdots]$ sums over Matsubara frequency and sublattice degrees of freedom. We have

$$
\begin{aligned}
\tilde{\Pi}_{\alpha \beta}(\mathbf{k}, \mathbf{q}, i \Omega)= & \frac{-i \Omega}{\Omega^{2}+\left(\left|\overrightarrow{\mathcal{F}}_{\mathbf{k}}\right|+\left|\overrightarrow{\mathcal{F}}_{\mathbf{k}-\mathbf{q}}\right|\right)^{2}}\left\{-\operatorname{Tr}\left[\tau^{\alpha} \tau^{\beta} \frac{\overrightarrow{\mathcal{F}}_{\mathbf{k}-\mathbf{q}} \cdot \vec{\tau}}{2\left|\overrightarrow{\mathcal{F}}_{\mathbf{k}-\mathbf{q}}\right|}\right]+\operatorname{Tr}\left[\tau^{\alpha} \frac{\overrightarrow{\mathcal{F}}_{\mathbf{k}} \cdot \vec{\tau}}{2\left|\overrightarrow{\mathcal{F}}_{\mathbf{k}}\right|} \tau^{\beta}\right]\right\} \\
& +\frac{-\left(\left|\overrightarrow{\mathcal{F}}_{\mathbf{k}}\right|+\left|\overrightarrow{\mathcal{F}}_{\mathbf{k}-\mathbf{q}}\right|\right)}{\Omega^{2}+\left(\left|\overrightarrow{\mathcal{F}}_{\mathbf{k}}\right|+\left|\overrightarrow{\mathcal{F}}_{\mathbf{k}-\mathbf{q}}\right|\right)^{2}}\left\{\frac{1}{2} \operatorname{Tr}\left[\tau^{\alpha} \tau^{\beta}\right]-\frac{1}{2} \operatorname{Tr}\left[\tau^{\alpha} \frac{\overrightarrow{\mathcal{F}}_{\mathbf{k}} \cdot \vec{\tau}}{2\left|\overrightarrow{\mathcal{F}}_{\mathbf{k}}\right|} \tau^{\beta} \frac{\overrightarrow{\mathcal{F}}_{\mathbf{k}-\mathbf{q}} \cdot \vec{\tau}}{2\left|\overrightarrow{\mathcal{F}}_{\mathbf{k}-\mathbf{q}}\right|}\right]\right\} .
\end{aligned}
$$

The first line (hereafter referred to as contribution I) and the second line (contribution II) have different physical meanings. Contribution I contains a term linear in $\Omega$ and is odd under time reversal, i.e., $\Omega \rightarrow-\Omega$ (in terms of both Matsubara frequency and time-ordered response in real frequency). Physically, this means that I contributes to the low-energy effective phonon action when time-reversal symmetry is broken. Contribution II renormalizes the real part of the phonon propagator, e.g., the sound velocity, which is a small effect and thus we do not consider it further.

The above observations are manifest by computing $\Pi_{\mathrm{ph}}^{\perp \|}(\mathbf{q}, i \Omega)$ using the vertex functions $\hat{\lambda}_{\mathbf{q}, \mathbf{k}}^{\|(\perp)}$ and summing $\mathbf{k}$ over the whole Brillouin zone. The MFPh coupling vertices originating from the time-reversal symmetry-breaking spin Hamiltonian are ignored because they are smaller than those in Eq. (27) by a factor of $\kappa / J_{K} \sim\left(h / J_{K}\right)^{3}$. Computing the contribution to $\Pi_{\mathrm{ph}}^{\perp \|}(\mathbf{q}, i \Omega)$ from part I in Eq. (50), which leads to the Hall viscosity term, we get

$$
\begin{aligned}
\Pi_{\mathrm{ph}}^{\perp \|}(\mathbf{q}, i \Omega)= & -\operatorname{Tr}\left[\hat{\lambda}_{\mathbf{q}, \mathbf{k}}^{\|} \mathcal{G}(\mathbf{k}, i \omega) \hat{\lambda}_{\mathbf{q}, \mathbf{k}}^{\perp} \mathcal{G}(\mathbf{k}-\mathbf{q}, i(\omega+\Omega))\right] \\
= & -q^{2} \operatorname{Tr}\left[\left(\lambda_{x, \mathbf{k}, \mathbf{q}}^{\|} \tau^{x}+\lambda_{y, \mathbf{k}, \mathbf{q}}^{\|} \tau^{y}\right) \mathcal{G}(\mathbf{k}, i \omega)\right. \\
& \left.\times\left(\lambda_{x, \mathbf{k}, \mathbf{q}}^{\perp} \tau^{x}+\lambda_{y, \mathbf{k}, \mathbf{q}}^{\perp} \tau^{y}\right) \mathcal{G}(\mathbf{k}-\mathbf{q}, i(\omega+\Omega))\right] \\
= & 2 q^{2} \Omega \int \frac{d^{2} k}{\mathcal{A}_{\mathrm{BZ}}} \frac{1}{\Omega^{2}+4\left|\overrightarrow{\mathcal{F}}_{\mathbf{k}}\right|^{2}} \frac{\Delta_{\mathbf{k}}}{\left|\overrightarrow{\mathcal{F}}_{\mathbf{k}}\right|} \\
& \times\left(\lambda_{x, \mathbf{k}, \mathbf{q}}^{\|} \lambda_{y, \mathbf{k}, \mathbf{q}}^{\perp}-\lambda_{y, \mathbf{k}, \mathbf{q}}^{\|} \lambda_{x, \mathbf{k}, \mathbf{q}}^{\perp}\right)
\end{aligned}
$$

where we have used $\operatorname{Tr}\left[\tau^{\alpha} \tau^{\beta} \tau^{\gamma}\right]=2 i \epsilon^{\alpha \beta \gamma}$ and defined $\lambda_{x, y}^{\|(\perp)}$ as the $\tau^{x, y}$ components of the MFPh coupling vertices $\hat{\lambda}_{\mathbf{q}, \mathbf{k}}^{\|(\perp)}$ :

$$
\begin{aligned}
& \hat{\lambda}_{x, \mathbf{q}, \mathbf{k}}^{\|}=\frac{i \lambda_{A_{1}}}{2} q f_{\mathbf{k}}^{\prime \prime}+\frac{i \lambda_{E_{2}}}{2} q\left(\cos 2 \theta_{q} f_{1, \mathbf{k}}^{\prime \prime}+\sin 2 \theta_{q} f_{2, \mathbf{k}}^{\prime \prime}\right), \\
& \hat{\lambda}_{y, \mathbf{q}, \mathbf{k}}^{\|}=\frac{i \lambda_{A_{1}}}{2} q f_{\mathbf{k}}^{\prime}+\frac{i \lambda_{E_{2}}}{2} q\left(\cos 2 \theta_{q} f_{1, \mathbf{k}}^{\prime}+\sin 2 \theta_{q} f_{2, \mathbf{k}}^{\prime}\right), \\
& \hat{\lambda}_{x, \mathbf{q}, \mathbf{k}}^{\perp}=\frac{i \lambda_{E_{2}}}{2} q\left(-\sin 2 \theta_{q} f_{1, \mathbf{k}}^{\prime \prime}+\cos 2 \theta_{q} f_{2, \mathbf{k}}^{\prime \prime}\right), \\
& \hat{\lambda}_{y, \mathbf{q}, \mathbf{k}}^{\perp}=\frac{i \lambda_{E_{2}}}{2} q\left(-\sin 2 \theta_{q} f_{1, \mathbf{k}}^{\prime}+\cos 2 \theta_{q} f_{2, \mathbf{k}}^{\prime}\right) .
\end{aligned}
$$

Note that from Eq. (51) one clearly sees that the contribution to $\Pi_{\mathrm{ph}}^{\mu \nu}(\mathbf{q}, i \Omega)$ from part I vanishes for $\mu=v$ and that $\Pi_{\mathrm{ph}}^{\| \perp}=$ $-\Pi_{\mathrm{ph}}^{\perp \|}$. Furthermore, since $\Delta_{\mathbf{k}} \propto \kappa$, the contribution from Eq. (51) only exists when time-reversal symmetry is broken (i.e., when $\kappa \neq 0$ ).

The main contribution to the momentum integration is near the Dirac points $( \pm K)$. Linearizing the spectrum near the Dirac points gives $\left|\overrightarrow{\mathcal{F}}_{ \pm K+\mathbf{k}}\right|=\left\{\mp 3 J_{K} k_{y},-3 J_{K} k_{x}, 6 \sqrt{3} \kappa\right\}$ and Eq. (51) becomes

$$
\Pi_{\mathrm{ph}}^{\perp \|}(\mathbf{q}, i \Omega) \approx-q^{2} \Omega \frac{\lambda_{E_{2}}^{2}}{J_{K}^{2}} \frac{3 \sqrt{3}}{4 \pi} \operatorname{sgn} \kappa .
$$

We note that the result is "quantized" in the sense that it does not depend on microscopic details of the time-reversal symmetry-breaking perturbation, i.e., the value of $\kappa$, but only on its sign. In fact, one can show that it is essentially the 


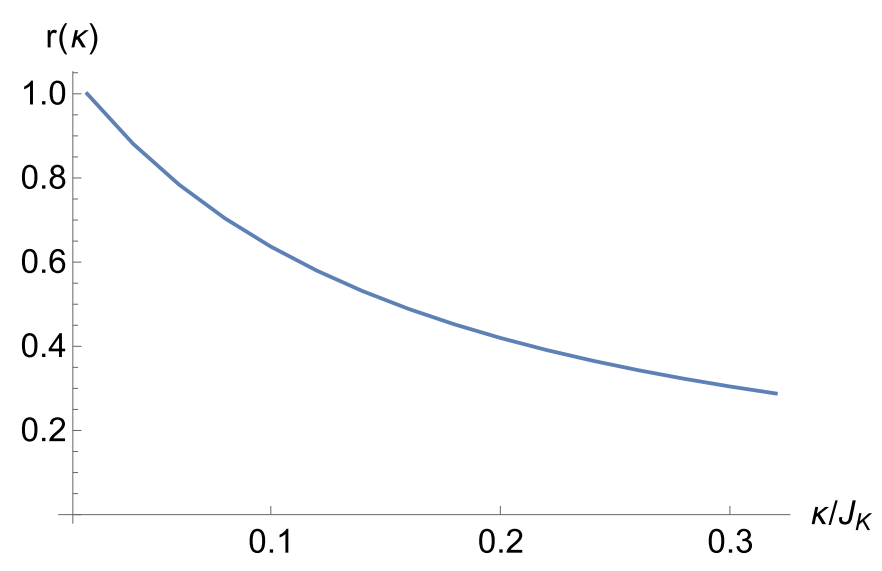

FIG. 3. Plot of $r(\kappa)$ as a function of the dimensionless ratio $\kappa / J_{K}$.

same setting to compute Hall conductivity of the anomalous quantum Hall effect when the MFPh vertex is constrained to near $\pm K$ [43]. On the other hand, the analogy breaks down beyond the linear approximation of the Majorana-fermion spectrum, since the high-energy Majorana fermions also contribute to the Hall viscosity. After integrating over the whole Brillouin zone, we find that the numerical prefactor depends on the magnitude of $\kappa$, i.e., on the magnitude of the Dirac mass.

In the following, we define the result found in Eq. (53) as $\Pi_{\mathrm{ph}}^{\perp \|}(q, i \Omega)_{\kappa \rightarrow 0}$, since in the limit $|\kappa| / J_{K} \rightarrow 0^{+}$, Eq. (53) is recovered. This is because the details of the Majoranafermion spectrum are not relevant in this limit, so the result should be the same as that obtained from the approximation of linearized spectrum. The polarization bubble at finite $\kappa$ can be expressed as $\Pi_{\mathrm{ph}}^{\perp \|}(q, i \Omega)_{\kappa}=r(\kappa) \Pi_{\mathrm{ph}}^{\perp \|}(q, i \Omega)_{\kappa \rightarrow 0}$, where the numerically evaluated coefficient $r(\kappa)$ is shown in Fig. 3. We note from the numerical calculation that only the $\lambda_{E_{2}}^{2}$ term in Eq. (51) contributes to the Hall viscosity coefficient.

The time-ordered phonon polarization bubble in real frequency can be obtained by analytic continuation. We have

$$
\operatorname{Im} \Pi_{\mathrm{ph}}^{\perp \|}(\mathbf{q}, \Omega)_{\kappa}=q^{2} \Omega \frac{\lambda_{E_{2}}^{2}}{J_{K}^{2}} \operatorname{sgn} \kappa \frac{3 \sqrt{3}}{4 \pi} r(\kappa) .
$$

Here we need to stress that while both Eq. (54), leading to the Hall viscosity, and the imaginary part of the real-time-ordered phonon polarization bubble associated with the sound attenuation as we found in Eqs. (43) and (44) are linear in $\Omega$, they are fundamentally different, i.e., the former is nondissipative and the latter dissipative. As was emphasized in our microscopic derivation, this difference comes from the distinct origins of the microscopic processes contributing to the imaginary part: One comes from the poles of the polarization bubble and the other from the numerator $\sim \operatorname{Tr}\left[\tau^{x} \tau^{y} \tau^{z}\right]$. From a phenomenological perspective, the difference comes from the fact that the imaginary part of the time-ordered polarization bubble associated with dissipation is even under $\Omega \rightarrow-\Omega$ due to causality, i.e., $\sim|\Omega|$.

On the other hand, the imaginary part of the time-ordered polarization bubble associated with the Hall viscosity is odd under $\Omega \rightarrow-\Omega$, i.e., $\sim \Omega$. This is consistent with the causal- ity constraint because the Hall viscosity term only appears in the off-diagonal component of the phonon polarization $\Pi_{\mathrm{ph}}^{\mu \nu}(\mathbf{q}, \Omega)$.

The Hall viscosity coefficient $\eta_{H}$ in Eq. (16) can now be related to the phonon polarization bubble by expressing Eq. (32) in terms of

$$
\Pi_{\mathrm{ph}}^{\mu \nu}(\mathbf{q}, \Omega)=\frac{-i}{2 !} \int d t e^{i \Omega t}\left\langle T \frac{\partial H_{c}}{\partial \tilde{u}_{\mathbf{q}, \mu}}(t) \frac{\partial H_{c}}{\partial \tilde{u}_{-\mathbf{q}, v}}(0)\right\rangle
$$

where we recall that $H_{c}$ is the spin-lattice coupling Hamiltonian. Comparing with the expression in Eq. (16), we find the Hall viscosity coefficient to be equal to

$$
\eta_{H}=-\frac{1}{\ell_{a}^{2}} \frac{\lambda_{E_{2}}^{2}}{J_{K}^{2}} \operatorname{sgn} \kappa \frac{3 \sqrt{3}}{2} r(\kappa) .
$$

Finally, we also note that the Hall viscosity term may be the starting point to obtain the phonon thermal Hall conductivity, which is finite and of order $\sim T^{3}[43,51]$.

\section{B. Renormalization of the phonon spectrum}

Next we discuss the renormalization of the phonon spectrum due to $\Pi_{\mathrm{ph}}^{\| \perp}(\mathbf{q}, \Omega)$ that mixes the longitudinal and transverse phonon modes. For brevity in the expression, we write Eq. (54) as $\operatorname{Im} \Pi_{\mathrm{ph}}^{\perp \|}(\mathbf{q}, \Omega)_{\kappa}=q^{2} \Omega g(\kappa)$, where

$$
g(\kappa) \equiv \frac{\lambda_{E_{2}}^{2}}{J_{K}^{2}} \frac{3 \sqrt{3}}{4 \pi} \operatorname{sgn} \kappa r(\kappa)
$$

The renormalized phonon propagator can be expressed in matrix form as

$D^{-1}(\mathbf{q}, \Omega)=-\rho \delta_{V}\left(\begin{array}{cc}\Omega^{2}-\left(v_{s}^{\|} q\right)^{2} & i q^{2} \Omega g(\kappa) /\left(\rho \delta_{V}\right) \\ -i q^{2} \Omega g(\kappa) /\left(\rho \delta_{V}\right) & \Omega^{2}-\left(v_{s}^{\perp} q\right)^{2}\end{array}\right)$.

To the leading order in $g(\kappa)$, the renormalized spectrum is given by

$$
\begin{aligned}
\Omega_{\mu, \mathbf{q}} & =v_{s}^{\mu} q \sqrt{1+q^{2} \frac{\left[g(\kappa) / \rho \delta_{V}\right]^{2}}{\left(v_{s}^{\mu}\right)^{2}-\left(v_{s}^{v}\right)^{2}}} \\
& \sim v_{s}^{\mu} q\left[1+\frac{1}{2} q^{2} \frac{\left[g(\kappa) / \rho \delta_{V}\right]^{2}}{\left(v_{s}^{\mu}\right)^{2}-\left(v_{s}^{v}\right)^{2}}\right] .
\end{aligned}
$$

Note that the correction from the $q^{3}$ term changes sign for the longitudinal and transverse phonon modes, so the spectrum bends upward (downward) for the longitudinal (transverse) mode [see Fig. 1(c)]. To determine the energy scale (relative to the Kitaev interaction $J_{K}$ ) when the deviation from the linear spectrum becomes prominent, the second term inside the large square brackets on the right-hand side of Eq. (59) can be written as

$$
\begin{aligned}
\frac{1}{2} q^{2} & \frac{\left(g(\kappa) / \rho \delta_{V}\right)^{2}}{\left(v_{s}^{\mu}\right)^{2}-\left(v_{s}^{v}\right)^{2}} \\
\quad= & \frac{1}{2}\left(\frac{v_{s}^{\mu} q}{J_{K}}\right)^{2}\left(\frac{\lambda_{E_{2}}^{2}}{J_{K} C_{\mu} \delta_{V}}\right)^{2}\left(\frac{\left(v_{s}^{\mu}\right)^{2}}{\left(v_{s}^{\mu}\right)^{2}-\left(v_{s}^{v}\right)^{2}}\right)\left(\frac{3 \sqrt{3}}{4 \pi} r(\kappa)\right)^{2},
\end{aligned}
$$


where the relation between the sound velocity, the mass density, and the components of the elastic modulus tensor was defined in Eq. (12) and $C_{\|}=C_{1}+C_{2}$ and $C_{\perp}=C_{2}$. It is straightforward to see that each term in large parentheses in Eq. (60) represents a dimensionless quantity. Thus, when Eq. (60) is comparable to 1 , the cubic term in $q$ becomes important. Therefore, when $v_{s}^{\perp}<v_{s}^{\|}$, the mixing shifts the transverse mode spectrum downward and the longitudinal mode spectrum upward at a characteristic scale

$$
\begin{aligned}
\delta_{c} & =\left(\frac{v_{s}^{\|} q}{J_{K}}\right)_{c} \sim \frac{J_{K} C_{\|} \delta_{V}}{\lambda_{E_{2}}^{2}} \frac{\sqrt{\left(v_{s}^{\|}\right)^{2}-\left(v_{s}^{\perp}\right)^{2}}}{v_{s}^{\|}} \\
& =\frac{J_{K} \sqrt{C_{\|}\left(C_{\|}-C_{\perp}\right)} \delta_{V}}{\lambda_{E_{2}}^{2}} .
\end{aligned}
$$

We note that in general $C \delta_{V}$ is a large energy scale of order of $\mathrm{eV}$, so large spin-lattice coupling is necessary to see appreciable change of the phonon spectrum due to the time-reversal breaking $\kappa$ term. However, more knowledge of the energy scales associated with $\lambda_{E_{2}}$ and $\sqrt{C_{\|}\left(C_{\|}-C_{\perp}\right)} \delta_{V}$ in Kitaev materials is needed to have a quantitative estimate of $\delta_{c}$.

\section{SUMMARY AND DISCUSSION}

In this work we have studied the effects of the spin-lattice coupling on the acoustic phonon dynamics in the Kitaev spin liquid and obtained various observables, such as the sound attenuation $\alpha_{s}$, the Hall viscosity coefficient $\eta_{H}$, and the renormalized 2D acoustic phonon spectrum [see Eq. (59)]. We suggest that if measured, these observable effects can be used as potential probes of spin fractionalization in the Kitaev materials.

We demonstrated that these observables can be obtained from the phonon polarization bubble. To compute the bubble, a microscopic low-energy effective spin-lattice coupling was derived and a diagrammatic computation procedure was formulated in terms of the matter Majorana fermions and the acoustic phonons. We first showed that the sound attenuation comes from the phonon scattering off the Majorana fermions. Due to the temperature dependence of the scattering phase space, $\alpha_{s} \propto T$, which distinguishes from the attenuation in other interaction channels, such as proportional to $T^{3}$ due to anharmonic phonon interactions. We also showed that $\alpha_{s}$ is highly anisotropic with an angular modulation proportional to $1 \pm \cos 6 \theta_{q}$, which should be attributed to the combined effects of the spin-lattice coupling and the low-energy Dirac spectrum of the Majorana fermions. We then computed the Hall viscosity coefficient of the phonon effective action, which is nonzero when time-reversal symmetry is broken. Different from electron Hall fluids, the Hall viscosity coefficient is not quantized. We found two contributions: one that is nonperturbative and resembles the quantized Hall conductance and another that is perturbative in the time-reversal breaking term $\kappa$. The Hall viscosity coefficient may be probed indirectly from the phonon spectrum, where it is manifested as a deviation of the phonon dispersion from linear to nonlinear near a characteristic momentum $q_{c}$, which depends on the strength of the spin-lattice coupling [see Eq. (61)]. Of course, such a deviation is generically expected for large enough momenta, where our long-wavelength approximation breaks down. Our key point is that this scale $q_{c}$ where the deviation from linear to nonlinear takes place is only present in the presence of an applied magnetic field, i.e., when time-reversal symmetry is broken. To obtain a quantitative estimate of the sound attenuation coefficient $\alpha_{s}$ and characteristic momentum $q_{c}$, we call for studies of the spin-lattice coupling and elastic modulus tensor of Kitaev materials from experiments and $a b$ initio studies.

As a final remark, we would like to emphasize that only in the temperature range $T<\Delta_{\text {flux }}$, which we focused in this work, can the thermal flux excitations be ignored. On the other hand, the key qualitative features we found, such as the linear in $T$ dependence and angular anisotropy of the sound attenuation coefficient, should remain valid up to a higher-temperature cutoff than the one set by the flux gap. Understanding the effects of spin-lattice coupling in the temperature range beyond the constraint set by $\Delta_{\text {flux }}$ would be highly desirable [40] and we leave it for future work.

\section{ACKNOWLEDGMENTS}

We thank Sananda Biswas, Wolfram Brenig, Fiona Burnell, Itamar Kimchi, Sai Mu, Lucile Savary, Xuzhe Ying, Roser Valenti, and Sergei Zherlitsyn for helpful discussions. M.Y. is grateful for support from the University of Minnesota through a Louise Dosdall Fellowship, the Army Research Office MURI Grant No. ARO W911NF-16-1-0361 (Floquet engineering and metastable states), and the National Science Foundation under Grant No. NSF 1748958 from UC Santa Barbara. R.M.F. was supported by the U.S. Department of Energy, Office of Science, Basic Energy Sciences, Materials Sciences and Engineering Division, under Award No. DE-SC0020045. The work of N.B.P. was supported by the U.S. Department of Energy, Office of Science, Basic Energy Sciences under Award No. DE-SC0018056. N.B.P. also acknowledges the hospitality of the KITP and NSF Grant No. 1748958 .

\section{APPENDIX A: MAJORANA-FERMION PROPAGATOR}

In this Appendix we briefly review the procedure of obtaining the Majorana-fermion Green's function, i.e., Eq. (33). It can be done either by the diagonalization of the Hamiltonian (2) directly keeping track of the independent Majoranafermion modes or by expressing Majorana fermions in terms of complex fermions. In the following, we take the second approach and express $\left\{c_{\mathbf{r}, A}, c_{\mathbf{r}, B}\right\}$ in terms of complex fermions $\gamma_{\mathbf{r}}$ on the bond as $c_{\mathbf{r}, A}=\left(\gamma_{\mathbf{r}}+\gamma_{\mathbf{r}}^{\dagger}\right)$ and $c_{\mathbf{r}, B}=i\left(\gamma_{\mathbf{r}}-\gamma_{\mathbf{r}}^{\dagger}\right)$. Performing the Fourier transformation to the momentum space $\gamma_{\mathbf{k}}=\frac{1}{\sqrt{N}} \sum \gamma_{\mathbf{r}} e^{-i \mathbf{k} \cdot \mathbf{r}}$, we can relate $\gamma_{\mathbf{k}}$ and $\gamma_{\mathbf{k}}^{\dagger}$ to $a_{\mathbf{k}}$ and $b_{\mathbf{k}}$ as $\gamma_{\mathbf{k}}=\frac{1}{\sqrt{2}}\left(a_{\mathbf{k}}-i b_{\mathbf{k}}\right)$ and $\gamma_{\mathbf{k}}^{\dagger}=\frac{1}{\sqrt{2}}\left(a_{-\mathbf{k}}+i b_{-\mathbf{k}}\right)$ and thus obtain

$$
\begin{aligned}
\tilde{H}_{s} & =\frac{1}{2} \sum_{\mathbf{k}} \psi_{-\mathbf{k}}^{T}\left(-\tau_{x} \operatorname{Im} f_{\mathbf{k}}-\tau_{y} \operatorname{Re} f_{\mathbf{k}}+\tau_{z} \Delta_{\mathbf{k}}\right) \psi_{\mathbf{k}} \\
& \rightarrow \frac{1}{2} \sum_{\mathbf{k}}\left(\gamma_{\mathbf{k}}^{\dagger}, \gamma_{-\mathbf{k}}\right)\left(\begin{array}{cc}
-f_{\mathbf{k}}^{\prime} & i f_{\mathbf{k}}^{\prime \prime}+\Delta_{\mathbf{k}} \\
-i f_{\mathbf{k}}^{\prime \prime} k+\Delta_{\mathbf{k}} & f_{\mathbf{k}}^{\prime}
\end{array}\right)\left(\begin{array}{c}
\gamma_{\mathbf{k}} \\
\gamma_{-\mathbf{k}}^{\dagger}
\end{array}\right),
\end{aligned}
$$


which can be diagonalized through Bogoliubov transformation. The Hamiltonian in terms of Bogoliubov fermions $\beta_{\mathbf{k}}$ is

$$
\tilde{H}_{s}=\frac{1}{2} \sum_{\mathbf{k}}\left(\beta_{\mathbf{k}}^{\dagger}, \beta_{-\mathbf{k}}\right)\left(\begin{array}{cc}
\left|\overrightarrow{\mathcal{F}}_{\mathbf{k}}\right| & 0 \\
0 & -\left|\overrightarrow{\mathcal{F}}_{\mathbf{k}}\right|
\end{array}\right)\left(\begin{array}{c}
\beta_{\mathbf{k}} \\
\beta_{-\mathbf{k}}^{\dagger}
\end{array}\right),
$$

from which we find $-i\left\langle T \beta_{\mathbf{k}}^{\dagger} \beta_{\mathbf{k}}\right\rangle_{\omega}=\frac{1}{\omega+\left|\overrightarrow{\mathcal{F}}_{\mathbf{k}}\right|-i \delta} \quad$ and $-i\left\langle T \beta_{k} \beta_{\mathbf{k}}^{\dagger}\right\rangle_{\omega}=\frac{1}{\omega-\left|\overrightarrow{\mathcal{F}}_{\mathbf{k}}\right|+i \delta}$. Using the transformation between $\psi_{\mathbf{k}}$ and $\beta_{\mathbf{k}}$, we obtain Eq. (33).

\section{APPENDIX B: RELATIONSHIP BETWEEN THE SOUND ATTENUATION COEFFICIENT $\alpha_{s}$ AND THE PHONON POLARIZATION BUBBLE}

To derive Eq. (39), we consider first the attenuation coefficient of a one-dimensional system in which the sound wave propagates in a given direction. We start with the lossy sound-wave equation [38]

$$
v_{s}^{2}\left(1+\tau_{s} \frac{\partial}{\partial t}\right) \nabla^{2} \mathbf{u}=\frac{\partial^{2}}{\partial t^{2}} \mathbf{u},
$$

where $\tau_{s}$ is the sound-wave relaxation time. With a plane-wave ansatz for $\mathbf{u}(x, t)=\mathbf{u}_{0} e^{i(\Omega t-q x)}$, we find that

$$
q=\frac{\Omega}{v_{s}} \frac{1}{\sqrt{1+i \Omega \tau_{s}}} \approx \frac{\Omega}{v_{s}}\left(1-\frac{i \Omega \tau_{s}}{2}\right) .
$$

Then the lossy sound wave becomes

$$
\mathbf{u}(x, t) \approx \exp \left[i\left(\Omega t-\frac{\Omega}{v_{s}} x\right)\right] \exp \left(-\frac{\Omega^{2} \tau_{s}}{2 v_{s}} x\right)
$$

and the sound attenuation coefficient can be simply written as

$$
\alpha_{s}=\frac{\Omega^{2} \tau_{s}}{2 v_{s}} .
$$

Following Eq. (B1), the phonon propagator can be expressed as

$$
D^{-1}(q, \Omega)=\rho \delta_{V}\left(\Omega^{2}-v_{s}^{2} q^{2}-i \Omega \tau_{s} v_{s}^{2} q^{2}\right) .
$$

On the other hand, the inverse of the renormalized phonon propagator (34) can be written as

$$
D^{-1}(q, \Omega)=\rho \delta_{V}\left(\Omega^{2}-v_{s}^{2} q^{2}\right)+i \operatorname{Im} \Pi_{\mathrm{ph}}(q, \Omega),
$$

where the real part of the phonon self-energy $\operatorname{Re} \hat{\Pi}_{\mathrm{ph}}(q, \Omega)$ has been absorbed into the renormalization of sound velocity $v_{s}$. Equating Eqs. (B6) and (B5) allows us to relate the lifetime $\tau_{s}$ to the imaginary part of phonon self-energy as

$$
\tau_{s}=-\frac{1}{\Omega v_{s}^{2} q^{2} \rho \delta_{V}} \operatorname{Im} \Pi_{\mathrm{ph}}(q, \Omega)
$$

and through this relate the sound attenuation coefficient with the imaginary part of phonon self-energy

$$
\alpha_{s}(q)=-\frac{1}{2 v_{s} \Omega_{q} \rho \delta_{V}} \operatorname{Im} \Pi_{\mathrm{ph}}\left(q, \Omega_{q}\right),
$$

where $\Omega_{q}=v_{s} q$. We note that $\operatorname{Im} \hat{\Pi}_{\mathrm{ph}} / \rho \delta_{V}$ is in units of energy squared and $\alpha_{s}(q)$ is in units of inverse distance. Equation (B8) can be straightforwardly generalized to higher dimensions by replacing the scalar $q$ with $\mathbf{q}$.

\section{APPENDIX C: TECHNICAL DETAILS OF THE EVALUATION OF THE SOUND ATTENUATION COEFFICIENT $\boldsymbol{\alpha}_{s}$}

As we discussed in Sec. VIA, when $T<\Delta_{\text {flux }} \ll J_{K}$ the spectrum of the Majorana fermions can be linearized near the Dirac points $\pm K$ and the MFPh vertices $\hat{\lambda}_{\mathbf{q}, \mathbf{k}}^{v}$ are constant in terms of $\mathbf{k}$. Thus, the momentum summation in Eq. (36) can be obtained by first replacing the true MFPh vertices with the Pauli matrices $\tau^{x, y}$, i.e., Eq. (42),

$$
\begin{aligned}
& \tilde{\Pi}_{\alpha \beta}(\mathbf{q}, \Omega)= \operatorname{Tr}\left[\tau^{\alpha} \mathcal{G}\left(\mathbf{k}, i \omega_{m}\right) \tau^{\beta} \mathcal{G}\left(\mathbf{k}-\mathbf{q}, i\left(\omega_{m}+\Omega_{n}\right)\right)\right]_{i \Omega \rightarrow \Omega+i \delta \operatorname{sgn} \Omega} \\
&= \sum_{s, s^{\prime}} \int \frac{d^{2} k}{\mathcal{A}_{\mathrm{BZ}}} \frac{1}{2} \frac{s \tanh \beta\left|\overrightarrow{\mathcal{F}}_{\mathbf{k}}\right| / 2-s^{\prime} \tanh \beta\left|\overrightarrow{\mathcal{F}}_{\mathbf{k}-\mathbf{q}}\right| / 2}{\Omega+s^{\prime}\left|\overrightarrow{\mathcal{F}}_{\mathbf{k}-\mathbf{q}}\right|-s\left|\overrightarrow{\mathcal{F}}_{\mathbf{k}}\right|+i \delta \operatorname{sgn} \Omega} \operatorname{Tr}\left[\tau^{\alpha}\left(\frac{I}{2}-s \frac{\overrightarrow{\mathcal{F}}_{\mathbf{k}} \cdot \vec{\tau}}{2\left|\overrightarrow{\mathcal{F}}_{\mathbf{k}}\right|}\right) \tau^{\beta}\left(\frac{I}{2}-s^{\prime} \frac{\overrightarrow{\mathcal{F}}_{\mathbf{k}-\mathbf{q}} \cdot \vec{\tau}}{2\left|\overrightarrow{\mathcal{F}}_{\mathbf{k}-\mathbf{q}}\right|}\right)\right] \\
&= \int \frac{d^{2} k}{\mathcal{A}_{\mathrm{BZ}}} \frac{1}{2}\left(\tanh \frac{\beta\left|\overrightarrow{\mathcal{F}}_{\mathbf{k}}\right|}{2}-\tanh \frac{\beta\left|\overrightarrow{\mathcal{F}}_{\mathbf{k}-\mathbf{q}}\right|}{2}\right)\left\{\frac{1}{\Omega+\left|\overrightarrow{\mathcal{F}}_{\mathbf{k}-\mathbf{q}}\right|-\left|\overrightarrow{\mathcal{F}}_{\mathbf{k}}\right|+i \delta \operatorname{sgn} \Omega} \operatorname{Tr}\left[\tau^{\alpha}\left(\frac{I}{2}-\frac{\overrightarrow{\mathcal{F}}_{\mathbf{k}} \cdot \vec{\tau}}{2\left|\overrightarrow{\mathcal{F}}_{\mathbf{k}}\right|}\right) \tau^{\beta}\left(\frac{I}{2}-\frac{\overrightarrow{\mathcal{F}}_{\mathbf{k}-\mathbf{q}} \cdot \vec{\tau}}{2\left|\overrightarrow{\mathcal{F}}_{\mathbf{k}-\mathbf{q}}\right|}\right)\right]\right. \\
&\left.-\frac{1}{\Omega-\left|\overrightarrow{\mathcal{F}}_{\mathbf{k}-\mathbf{q}}\right|+\left|\overrightarrow{\mathcal{F}}_{\mathbf{k}}\right|+i \delta \operatorname{sgn} \Omega} \operatorname{Tr}\left[\tau^{\alpha}\left(\frac{I}{2}+\frac{\overrightarrow{\mathcal{F}}_{\mathbf{k}} \cdot \vec{\tau}}{2\left|\overrightarrow{\mathcal{F}}_{\mathbf{k}}\right|}\right) \tau^{\beta}\left(\frac{I}{2}+\frac{\overrightarrow{\mathcal{F}}_{\mathbf{k}-\mathbf{q}} \cdot \vec{\tau}}{2\left|\overrightarrow{\mathcal{F}}_{\mathbf{k}-\mathbf{q}}\right|}\right)\right]\right\}, \\
&(\mathrm{C} 1)
\end{aligned}
$$

where $\alpha, \beta=x, y$ and $\mathcal{A}_{\mathrm{BZ}}=8 \pi^{2} / 3 \sqrt{3}$ is the area of the honeycomb lattice Brillouin zone. Expanding $\overrightarrow{\mathcal{F}}_{\mathbf{k}}$ and $\overrightarrow{\mathcal{F}}_{\mathbf{k}-\mathbf{q}}$ near the Dirac points $\pm K$, we find

$$
\begin{aligned}
\tilde{\Pi}_{\alpha \beta}(\mathbf{q}, \Omega)= & \int \frac{d^{2} k}{\mathcal{A}_{\mathrm{BZ}}} \frac{1}{2}\left(\tanh \frac{\beta\left|\overrightarrow{\mathcal{F}}_{ \pm K+\mathbf{k}}\right|}{2}-\tanh \frac{\beta\left|\overrightarrow{\mathcal{F}}_{ \pm K+\mathbf{k}-\mathbf{q}}\right|}{2}\right) \frac{1}{\Omega+\left|\overrightarrow{\mathcal{F}}_{ \pm K+\mathbf{k}-\mathbf{q}}\right|-\left|\overrightarrow{\mathcal{F}}_{ \pm K+\mathbf{k}}\right|+i \delta \operatorname{sgn} \Omega} \\
& \times\left\{\frac{1}{2} \operatorname{Tr}\left[\tau^{\alpha} \tau^{\beta}\right]+\operatorname{Tr}\left[\tau^{\alpha} \frac{\overrightarrow{\mathcal{F}}_{ \pm K+\mathbf{k}} \cdot \vec{\tau}}{2\left|\overrightarrow{\mathcal{F}}_{ \pm K+\mathbf{k}}\right|} \tau^{\beta} \frac{\overrightarrow{\mathcal{F}}_{ \pm K+\mathbf{k}-\mathbf{q}} \cdot \vec{\tau}}{2\left|\overrightarrow{\mathcal{F}}_{ \pm K+\mathbf{k}-\mathbf{q}}\right|}\right]+\operatorname{Tr}\left[\tau^{\alpha} \frac{\overrightarrow{\mathcal{F}}_{ \pm K-\mathbf{k}+\mathbf{q}} \cdot \vec{\tau}}{2\left|\overrightarrow{\mathcal{F}}_{ \pm K-\mathbf{k}+\mathbf{q}}\right|} \tau^{\beta} \frac{\overrightarrow{\mathcal{F}}_{ \pm K-\mathbf{k}} \cdot \vec{\tau}}{2\left|\overrightarrow{\mathcal{F}}_{ \pm K-\mathbf{k}}\right|}\right]\right\} .
\end{aligned}
$$


Using the identities for Pauli matrices, $\operatorname{Tr}\left[\tau^{\alpha} \tau^{\beta}\right]=2 \delta^{\alpha \beta}$ and $\operatorname{Tr}\left[\tau^{\alpha} \tau^{\beta} \tau^{\gamma} \tau^{\delta}\right]=2\left(\delta^{\alpha \beta} \delta^{\gamma \delta}-\delta^{\alpha \gamma} \delta^{\beta \delta}+\delta^{\alpha \delta} \delta^{\beta \gamma}\right)$, we find that the second line for $\tilde{\Pi}^{\alpha \beta}$ can be simplified and we get

$$
\begin{aligned}
& \tilde{\Pi}_{x x}(\mathbf{q}, \Omega) \sim\left\{1+\frac{\left.\mathcal{F}_{ \pm K+\mathbf{k}}^{x} \mathcal{F}_{ \pm K+\mathbf{k}-\mathbf{q}}^{x}-\mathcal{F}_{ \pm K+\mathbf{k}}^{y} \mathcal{F}_{ \pm K+\mathbf{k}-\mathbf{q}}^{y}-\frac{\mathcal{F}_{ \pm K+\mathbf{k}}^{z} \mathcal{F}_{ \pm K+\mathbf{k}-\mathbf{q}}^{z}}{\left|\overrightarrow{\mathcal{F}}_{ \pm K+\mathbf{k}}\right|\left|\overrightarrow{\mathcal{F}}_{ \pm K+\mathbf{k}-\mathbf{q}}\right|}\right\}}{\left|\overrightarrow{\mathcal{F}}_{ \pm K+\mathbf{k}}\right|\left|\overrightarrow{\mathcal{F}}_{ \pm K+\mathbf{k}-\mathbf{q}}\right|}-\frac{\mathcal{F}_{ \pm K+\mathbf{k}}^{z} \mathcal{F}_{ \pm K+\mathbf{k}-\mathbf{q}}^{z}}{\left|\overrightarrow{\mathcal{F}}_{ \pm K+\mathbf{k}}\right|\left|\overrightarrow{\mathcal{F}}_{ \pm K+\mathbf{k}-\mathbf{q}}\right|}\right\} \\
& \tilde{\Pi}_{y y}(\mathbf{q}, \Omega) \sim\left\{1-\frac{\mathcal{F}_{ \pm K+\mathbf{k}}^{x} \mathcal{F}_{ \pm K+\mathbf{k}-\mathbf{q}}^{x}-\mathcal{F}_{ \pm K+\mathbf{k}}^{y} \mathcal{F}_{ \pm K+\mathbf{k}-\mathbf{q}}^{y}}{\left|\overrightarrow{\mathcal{F}}_{ \pm K+\mathbf{k}}\right|\left|\overrightarrow{\mathcal{F}}_{ \pm K+\mathbf{k}-\mathbf{q}}\right|}\right. \\
& \tilde{\Pi}_{x y}(\mathbf{q}, \Omega) \sim\left\{\frac{\mathcal{F}_{ \pm K+\mathbf{k}}^{x} \mathcal{F}_{ \pm K+\mathbf{k}-\mathbf{q}}^{y}+\mathcal{F}_{ \pm K+\mathbf{k}}^{y} \mathcal{F}_{ \pm K+\mathbf{k}-\mathbf{q}}^{x}}{\left|\overrightarrow{\mathcal{F}}_{ \pm K+\mathbf{k}}\right|\left|\overrightarrow{\mathcal{F}}_{ \pm K+\mathbf{k}-\mathbf{q}}\right|}\right\} .
\end{aligned}
$$

The diagonal components of $\operatorname{Im} \Pi_{\mathrm{ph}}^{v \nu}(\mathbf{q}, \Omega)$ in terms of $\tilde{\Pi}_{\alpha \beta}$ are then given by

$$
\begin{aligned}
& \Pi^{\|\|}(\mathbf{q}, \Omega)=9 \lambda_{E_{2}}^{2} q^{2}\left[\sin ^{2} 2 \theta_{q} \tilde{\Pi}_{x x}+\cos ^{2} 2 \theta_{q} \tilde{\Pi}_{y y}-\sin 2 \theta_{q} \cos 2 \theta_{q}\left(\tilde{\Pi}_{x y}+\tilde{\Pi}_{y x}\right)\right], \\
& \Pi^{\perp \perp}(\mathbf{q}, \Omega)=9 \lambda_{E_{2}}^{2} q^{2}\left[\cos ^{2} 2 \theta_{q} \tilde{\Pi}_{x x}+\sin ^{2} 2 \theta_{q} \tilde{\Pi}_{y y}+\sin 2 \theta_{q} \cos 2 \theta_{q}\left(\tilde{\Pi}_{x y}+\tilde{\Pi}_{y x}\right)\right] .
\end{aligned}
$$

These are formulas (45) and (46).

We first consider $\mathcal{F}^{z}=\Delta_{\mathbf{k}}=0$, which is the case when $\kappa=0$. Linearizing $\overrightarrow{\mathcal{F}}_{ \pm K+\mathbf{k}}$ near $K($ or $-K)$ as

$$
\overrightarrow{\mathcal{F}}_{K+\mathbf{k}}=\left|f_{K+\mathbf{k}}\right|\left\{-\sin \phi_{k},-\cos \phi_{k}, 0\right\}=v_{F} k\left\{-\sin \phi_{k},-\cos \phi_{k}, 0\right\},
$$

we find

$$
\begin{aligned}
& i \operatorname{Im} \Pi_{\mathrm{ph}}^{\|\|}(\mathbf{q}, \Omega)=-i 9 \lambda_{E_{2}}^{2} q^{2} \pi \int \frac{d^{2} k}{\mathcal{A}_{\mathrm{BZ}}} \operatorname{sgn} \Omega \delta\left(\Omega+\left|f_{K+\mathbf{k}-\mathbf{q}}\right|-\left|f_{K+\mathbf{k}}\right|\right)\left(\tanh \frac{\beta\left|f_{K+\mathbf{k}}\right|}{2}-\tanh \frac{\beta\left|f_{K+\mathbf{k}-\mathbf{q}}\right|}{2}\right) \\
& \times\left(1-\cos 4 \theta_{q} \frac{\mathcal{F}_{K+\mathbf{k}}^{x} \mathcal{F}_{K+\mathbf{k}-\mathbf{q}}^{x}-\mathcal{F}_{K+\mathbf{k}}^{y} \mathcal{F}_{K+\mathbf{k}-\mathbf{q}}^{y}}{\left|f_{K+\mathbf{k}}\right|\left|f_{K+\mathbf{k}-\mathbf{q}}\right|}-\sin 4 \theta_{q} \frac{\mathcal{F}_{K+\mathbf{k}}^{x} \mathcal{F}_{K+\mathbf{k}-\mathbf{q}}^{y}+\mathcal{F}_{K+\mathbf{k}}^{y} \mathcal{F}_{K+\mathbf{k}-\mathbf{q}}^{x}}{\left|f_{K+\mathbf{k}}\right|\left|f_{K+\mathbf{k}-\mathbf{q}}\right|}\right) \\
& =-i \operatorname{sgn} \Omega 9 \lambda_{E_{2}}^{2} q^{2} \pi\left(1-\cos 6 \theta_{q}\right) \int \frac{d^{2} k}{\mathcal{A}_{\mathrm{BZ}}} \delta\left(\Omega+\left|f_{K+\mathbf{k}-\mathbf{q}}\right|-\left|f_{K+\mathbf{k}}\right|\right)\left(\tanh \frac{\beta\left|f_{K+\mathbf{k}}\right|}{2}-\tanh \frac{\beta\left|f_{K+\mathbf{k}-\mathbf{q}}\right|}{2}\right) \\
& =-i \frac{18 \pi \lambda_{E_{2}}^{2} q|\Omega|}{v_{F}^{3} \mathcal{A}_{\mathrm{BZ}}} \beta\left(1-\cos 6 \theta_{q}\right) \int_{\epsilon>v_{F} q / 2} d \epsilon \frac{\epsilon}{\sqrt{4-\left(v_{F} q / \epsilon\right)^{2}}} \frac{1}{\cosh ^{2} \beta \epsilon / 2} \\
& =-i \frac{18 \pi \lambda_{E_{2}}^{2} q|\Omega|}{v_{F}^{3} \mathcal{A}_{\mathrm{BZ}}} T\left(1-\cos 6 \theta_{q}\right) \int_{v_{F} q \beta / 2}^{\infty} d x \frac{x^{2}}{\sqrt{4 x^{2}-\left(v_{F} q \beta\right)^{2}}} \frac{1}{\cosh ^{2} x / 2} \\
& \approx-i \frac{18 \pi \lambda_{E_{2}}^{2} q|\Omega|}{2 v_{F}^{3} \mathcal{A}_{\mathrm{BZ}}} T\left(1-\cos 6 \theta_{q}\right) \int_{0}^{\infty} d x \frac{x}{\cosh ^{2} x / 2}=-i \frac{36 \pi \lambda_{E_{2}}^{2} q|\Omega|}{v_{F}^{3} \mathcal{A}_{\mathrm{BZ}}} T \ln 2\left(1-\cos 6 \theta_{q}\right) .
\end{aligned}
$$

The imaginary part of $\operatorname{Im} \Pi_{\mathrm{ph}}^{\|\|}(\mathbf{q}, \Omega)$ comes from the pole in Eq. (41) using $\operatorname{Im} \frac{1}{\Omega+i \delta \operatorname{sgn} \Omega}=-i \pi \delta(\Omega) \operatorname{sgn} \Omega$. From the first to the second equation, we use

$$
\frac{\mathcal{F}_{K+\mathbf{k}}^{x} \mathcal{F}_{K+\mathbf{k}-\mathbf{q}}^{y}+\mathcal{F}_{K+\mathbf{k}}^{y} \mathcal{F}_{K+\mathbf{k}-\mathbf{q}}^{x}}{\left|f_{K+\mathbf{k}}\right|\left|f_{K+\mathbf{k}-\mathbf{q}}\right|}=-\sin 2 \theta_{q}, \quad \frac{\mathcal{F}_{K+\mathbf{k}}^{x} \mathcal{F}_{K+\mathbf{k}-\mathbf{q}}^{x}-\mathcal{F}_{K+\mathbf{k}}^{y} \mathcal{F}_{K+\mathbf{k}-\mathbf{q}}^{y}}{\left|f_{K+\mathbf{k}}\right|\left|f_{K+\mathbf{k}-\mathbf{q}}\right|}=\cos 2 \theta_{q} .
$$

From the penultimate to the last line, we require $v_{F} q \beta \ll 1\left(v_{F} q \ll T\right)$, which optimizes the decay, because otherwise the integrand is exponentially small as $\frac{1}{\cosh ^{2} x / 2} \ll 1$. Similarly, we find

$$
i \operatorname{Im} \Pi^{\perp \perp}(\mathbf{q}, \Omega)=-i \frac{36 \pi \lambda_{E_{2}}^{2} q|\Omega|}{v_{F}^{3} \mathcal{A}_{\mathrm{BZ}}} T \ln 2\left(1+\cos 6 \theta_{q}\right) .
$$

[1] P. Anderson, Mater. Res. Bull. 8, 153 (1973).

[2] X.-G. Wen, Phys. Rev. B 65, 165113 (2002).

[3] A. Kitaev, Ann. Phys. (NY) 321, 2 (2006).

[4] L. Balents, Nature (London) 464, 199 (2010).

[5] L. Savary and L. Balents, Rep. Prog. Phys. 80, 016502 (2017).
[6] G. Jackeli and G. Khaliullin, Phys. Rev. Lett. 102, 017205 (2009).

[7] S. Trebst, arXiv:1701.07056.

[8] S. M. Winter, Y. Li, H. O. Jeschke, and R. Valentí, Phys. Rev. B 93, 214431 (2016). 
[9] S. M. Winter, A. A. Tsirlin, M. Daghofer, J. van den Brink, Y. Singh, P. Gegenwart, and R. Valenti, J. Phys.: Condens. Matter 29, 493002 (2017).

[10] M. Hermanns, I. Kimchi, and J. Knolle, Annu. Rev. Condens. Matter Phys. 9, 17 (2018).

[11] H. Takagi, T. Takayama, G. Jackeli, G. Khaliullin, and S. E. Nagler, Nat. Rev. Phys. 1, 264 (2019).

[12] Y. Motome and J. Nasu, J. Phys. Soc. Jpn. 89, 012002 (2020).

[13] S. E. Nagler, D. A. Tennant, R. A. Cowley, T. G. Perring, and S. K. Satija, Phys. Rev. B 44, 12361 (1991).

[14] D. A. Tennant, T. G. Perring, R. A. Cowley, and S. E. Nagler, Phys. Rev. Lett. 70, 4003 (1993).

[15] J. Knolle, D. L. Kovrizhin, J. T. Chalker, and R. Moessner, Phys. Rev. B 92, 115127 (2015).

[16] J. Knolle, D. L. Kovrizhin, J. T. Chalker, and R. Moessner, Phys. Rev. Lett. 112, 207203 (2014).

[17] A. Banerjee, C. A. Bridges, J.-Q. Yan, A. A. Aczel, L. Li, M. B. Stone, G. E. Granroth, M. D. Lumsden, Y. Yiu, J. Knolle, S. Bhattacharjee, D. L. Kovrizhin, R. Moessner, D. A. Tennant, D. G. Mandrus, and S. E. Nagler, Nat. Mater. 15, 733 (2016).

[18] A. Banerjee, J. Yan, J. Knolle, C. A. Bridges, M. B. Stone, M. D. Lumsden, D. G. Mandrus, D. A. Tennant, R. Moessner, and S. E. Nagler, Science 356, 1055 (2017).

[19] W.-H. Ko, Z.-X. Liu, T.-K. Ng, and P. A. Lee, Phys. Rev. B 81, 024414 (2010).

[20] L. J. Sandilands, Y. Tian, K. W. Plumb, Y.-J. Kim, and K. S. Burch, Phys. Rev. Lett. 114, 147201 (2015).

[21] J. Knolle, G.-W. Chern, D. L. Kovrizhin, R. Moessner, and N. B. Perkins, Phys. Rev. Lett. 113, 187201 (2014).

[22] J. Nasu, J. Knolle, D. L. Kovrizhin, Y. Motome, and R. Moessner, Nat. Phys. 12, 912 (2016).

[23] I. Rousochatzakis, S. Kourtis, J. Knolle, R. Moessner, and N. B. Perkins, Phys. Rev. B 100, 045117 (2019).

[24] A. Sahasrabudhe, D. A. S. Kaib, S. Reschke, R. German, T. C. Koethe, J. Buhot, D. Kamenskyi, C. Hickey, P. Becker, V. Tsurkan, A. Loidl, S. H. Do, K. Y. Choi, M. Grueninger, S. M. Winter, Z. Wang, R. Valenti, and P. H. M. van Loosdrecht, Phys. Rev. B 101, 140410 (2020).

[25] D. Wulferding, Y. Choi, S.-H. Do, C. H. Lee, P. Lemmens, C. Faugeras, Y. Gallais, and K.-Y. Choi, Nat. Commun. 11, 1603 (2020).

[26] G. B. Halász, N. B. Perkins, and J. van den Brink, Phys. Rev. Lett. 117, 127203 (2016).

[27] G. B. Halász, S. Kourtis, J. Knolle, and N. B. Perkins, Phys. Rev. B 99, 184417 (2019).

[28] Z. Alpichshev, F. Mahmood, G. Cao, and N. Gedik, Phys. Rev. Lett. 114, 017203 (2015).

[29] Y. Wan and N. P. Armitage, Phys. Rev. Lett. 122, 257401 (2019).

[30] A. Pippard, Philos. Mag. 46, 1104 (1955).

[31] A. I. Akhiezer, M. I. Kaganov, and G. Y. Lyubarskyi, Sov. Phys. JETP 5, 685 (1957).
[32] E. I. Blount, Phys. Rev. 114, 418 (1959).

[33] T. Tsuneto, Phys. Rev. 121, 402 (1961).

[34] B. Batlogg, D. Bishop, B. Golding, C. M. Varma, Z. Fisk, J. L. Smith, and H. R. Ott, Phys. Rev. Lett. 55, 1319 (1985).

[35] H. Won and K. Maki, Phys. Rev. B 49, 1397 (1994).

[36] R. M. Fernandes, P. P. Orth, and J. Schmalian, Annu. Rev. Condens. Matter Phys. 10, 133 (2019).

[37] A. Kreisel, P. Kopietz, P. T. Cong, B. Wolf, and M. Lang, Phys. Rev. B 84, 024414 (2011).

[38] Y. Zhou and P. A. Lee, Phys. Rev. Lett. 106, 056402 (2011).

[39] M. Serbyn and P. A. Lee, Phys. Rev. B 87, 174424 (2013).

[40] A. Metavitsiadis and W. Brenig, Phys. Rev. B 101, 035103 (2020).

[41] Y. Kasahara, T. Ohnishi, Y. Mizukami, O. Tanaka, S. Ma, K. Sugii, N. Kurita, H. Tanaka, J. Nasu, Y. Motome, T. Shibauchi, and Y. Matsuda, Nature (London) 559, 227 (2018).

[42] M. Ye, G. B. Halász, L. Savary, and L. Balents, Phys. Rev. Lett. 121, 147201 (2018).

[43] Y. Vinkler-Aviv and A. Rosch, Phys. Rev. X 8, 031032 (2018).

[44] K. W. Plumb, J. P. Clancy, L. J. Sandilands, V. V. Shankar, Y. F. Hu, K. S. Burch, H.-Y. Kee, and Y.-J. Kim, Phys. Rev. B 90, 041112(R) (2014).

[45] S.-H. Do, S.-Y. Park, J. Yoshitake, J. Nasu, Y. Motome, Y. S. Kwon, D. T. Adroja, D. J. Voneshen, K. Kim, T.-H. Jang, J.-H. Park, K.-Y. Choi, and S. Ji, Nat. Phys. 13, 1079 (2017).

[46] D. Hirobe, M. Sato, Y. Shiomi, H. Tanaka, and E. Saitoh, Phys. Rev. B 95, 241112(R) (2017).

[47] T. O. Woodruff and H. Ehrenreich, Phys. Rev. 123, 1553 (1961).

[48] J. E. Avron, R. Seiler, and P. G. Zograf, Phys. Rev. Lett. 75, 697 (1995).

[49] M. Barkeshli, S. B. Chung, and X.-L. Qi, Phys. Rev. B 85, 245107 (2012).

[50] N. Read and E. H. Rezayi, Phys. Rev. B 84, 085316 (2011).

[51] T. Qin, J. Zhou, and J. Shi, Phys. Rev. B 86, 104305 (2012).

[52] G. D. Mahan, Many-Particle Physics (Plenum, New York, 1990).

[53] L. D. Landau, L. P. Pitaevskii, A. M. Kosevich, and E. M. Lifshitz, Theory of Elasticity, 3rd ed. (Butterworth-Heinemann, Oxford, 2012).

[54] M. S. Dresselhaus, G. Dresselhaus, and A. Jorio, Group Theory: Application to the Physics of Condensed Matter (Springer, Berlin, 2008).

[55] G. F. Koster, Properties of the Thirty-Two Point Groups (MIT Press, Cambridge, 1963).

[56] N. Ashcroft and N. Mermin, Solid State Physics (Holt, Rinehart and Winston, New York, 1976).

[57] A. Altland and B. D. Simons, Condensed Matter Field Theory, 2nd ed. (Cambridge University Press, Cambridge, 2010).

[58] N. Nagaosa, J. Sinova, S. Onoda, A. H. MacDonald, and N. P. Ong, Rev. Mod. Phys. 82, 1539 (2010). 